\title{
Combining states without scale hierarchies with ordered parton showers
}

\author{
Nadine Fischer $^{1, \mathrm{a}}$, Stefan Prestel $^{2}$ \\ ${ }^{1}$ School of Physics and Astronomy, Monash University, Clayton, VIC 3800, Australia \\ ${ }^{2}$ Fermi National Accelerator Laboratory, Batavia, IL 60510-0500, USA
}

Received: 26 June 2017 / Accepted: 23 August 2017 / Published online: 12 September 2017

(C) The Author(s) 2017. This article is an open access publication

\begin{abstract}
We present a parameter-free scheme to combine fixed-order multi-jet results with parton-shower evolution. The scheme produces jet cross sections with leading-order accuracy in the complete phase space of multiple emissions, resumming large logarithms when appropriate, while not arbitrarily enforcing ordering on momentum configurations beyond the reach of the parton-shower evolution equation. This requires the development of a matrix-element correction scheme for complex phase-spaces including ordering conditions as well as a systematic scale-setting procedure for unordered phase-space points. The resulting algorithm does not require a merging-scale parameter. We implement the new method in the VINCIA framework and compare to LHC data.
\end{abstract}

\section{Introduction}

High-energy physics in the era of the Large Hadron Collider relies on accurate calculations of Standard-Model scattering signatures-both to determine backgrounds when directly searching for new physics and to allow for setting indirect bounds by comparing measurements to precision calculations. Since measurements at the LHC are typically sensitive to the formation and evolution of jets, much attention has been devoted to calculating QCD corrections. This has led to exquisite dedicated high-precision calculations, and to the development of general schemes to overcome the limited applicability of individual fixed-order QCD calculations by combining multiple calculations into a single consistent result. To this end, modern General Purpose Event Generators [1-4] include a variety of complex matching [5-16] and merging [17-35] schemes.

A unified Standard-Model prediction that is applicable for precision measurements and new-physics searches alike

\footnotetext{
a e-mail: nadine.fischer@monash.edu
}

must naturally include particle configurations that probe very different aspects of the calculation. The optimal perturbative description of very different particle (and momentum) configurations can consequently vary significantly within one measurement, so that care must be taken to avoid applying specialized arguments outside of their region of validity. Otherwise, the accuracy of the calculation is in jeopardy and its uncertainty might be underestimated. For example, applying QCD reasoning to events without large hierarchies in the hardness of jets can lead to problematic effects [36].

Standard-model calculations at the LHC can somewhat artificially be categorized as focussing on momentum configurations with or without large scale (hardness) hierarchies between jets. Fixed-order QCD calculations are often appropriate for the latter, while the former require a resummation of large perturbative enhancements by means of evolution equations. Both approaches have complementary strengths and should be combined for a state-of-the-art calculation. It is crucial to avoid bias when constructing a single calculation that describes very different limits.

In this article, we design a new algorithm to combine multiple fixed-order calculations for different parton multiplicities with each other and with (parton-shower) resummation of large logarithmic enhancements. The aim of this combined calculation is to simultaneously describe up to $n$ hard, wellseparated partons with fixed-order matrix elements while retaining the jet evolution given by the parton shower. We enforce strict requirements on the new scheme to improve on previous ideas:

1. The introduction of new parameters into the calculation is avoided. This is especially important when the correlation with existing parameters is not obvious.

2. The method should provide a uniform accuracy over the complete phase space for one particle multiplicity. For now, this means that the rate of $n$ jets should be given 
with leading-order accuracy in QCD, irrespectively of the hardness of jets.

3. The method should be largely agnostic to parton-showerinspired arguments when configurations without large scale hierarchies are discussed.

The resulting method borrows concepts from the CKKW$\mathrm{L}$ method of merging matrix elements and parton showers [26-28], as well as from matrix-element correction schemes $[37,38]$. We provide a new solution to the treatment of phase-space regions beyond the reach of traditional shower evolution. Furthermore, we improve upon the structure of the combined calculation in the parton-shower region of soft and/or collinear emissions. Our new method consists of two main developments: the introduction (and implementation) of matrix-element corrections for ordered parton-shower evolution, and the definition of a general scale-setting prescription based on matrix elements for contributions without apparent scale hierarchies. The benefit of using matrix-element corrections for shower-like splitting sequences is that unitarity of fixed-order multi-jet cross sections is automatically guaranteed in these phase-space regions. This means that the inclusive rates for $n$ jets will be correctly described with fixed-order accuracy, without the need for explicit subtractions of negative weight, even if the rate for $n+1$ jets is also corrected with matrix elements. We will describe how the new method allows to achieve leading-order accuracy in QCD for multi-parton configurations. This establishes a baseline for future developments beyond leading-order QCD.

The new scheme relies on applying leading-order matrixelement corrections in phase-space regions that are accessible by a sequence of splittings ordered in a parton-shower evolution variable, supplemented with fixed-order results for configurations that cannot be reached by any such sequence. We will use the misnomer "shower configurations" for such states, and call states which cannot be reached by an ordered sequence of shower emissions "non-shower states".

A very brief introduction to the parton-shower formalism and the notation is established in Sect. 2. The new method to iteratively correct parton showers with matrix elements is described in detail in Sect. 3. The combination of this scheme of matrix-element corrections for ordered partonshower evolution with non-shower states is discussed in Sect. 4. An executive summary of the algorithm is given in Sect. 5 , followed by a discussion of the impact of combining parton-shower-like and non-shower phase-space regions at parton level. Then results and data comparisons are presented in Sect. 6 before we summarize and give an outlook in Sect. 7. Additional details about the smoothly ordered showers and "GKS" matrix-element corrections previously used in VINCIA are collected in Appendix A, while a thorough validation of new matrix-element corrections for ordered parton-shower evolution is given in Appendix B.

\section{Parton showers and matrix-element corrections}

To set the scene and establish notation, let us briefly review some parton-shower basics. We start by defining the effect of parton-shower evolution $[39,40]$ on an arbitrary observable $O$ (in the notation of [41]),

$$
\begin{aligned}
& \mathcal{F}_{\vec{a}}\left(\Phi_{n}, t, t^{\prime} ; O\right)=\mathcal{F}_{\vec{a}}\left(\Phi_{n}, t, t^{\prime}\right) O\left(\Phi_{n}\right) \\
& \quad+\int_{t}^{t^{\prime}} \frac{\mathrm{d} \bar{t}}{\bar{t}} \frac{\mathrm{d} \mathcal{F}_{\vec{a}}\left(\Phi_{n}, \bar{t}, t^{\prime}\right)}{\mathrm{d} \ln \bar{t}} \mathcal{F}_{\vec{a}^{\prime}}\left(\Phi_{n+1}^{\prime}, t, \bar{t} ; O\right),
\end{aligned}
$$

where $t \equiv t\left(\Phi_{n+1} / \Phi_{n}\right)$ is the shower evolution variable, and the shower generating functional $\mathcal{F}$ depends on the list of parton flavors $\vec{a}$, and the corresponding $n$-particle momentum configuration $\Phi_{n}$. Though not explicitly stated, any $n$ particle state contains an arbitrarily complicated Born state, $\Phi_{n} \equiv \Phi_{\mathrm{B}+n}$. The first term in Eq. (1) encodes the contribution from no resolvable shower emissions, while the second piece includes one or more emissions. The parton flavors $\vec{a}^{\prime}$ of the $(n+1)$-particle momentum configuration $\Phi_{n+1}$ include the resolved emission and the partons $\vec{a}$, with momenta changed according to the recoil prescription of the parton shower and flavor changes where applicable. The generating functional obeys the evolution equation

$$
\begin{aligned}
& \frac{\mathrm{d} \ln \mathcal{F}_{\vec{a}}\left(\Phi_{n}, t, \mu^{2}\right)}{\mathrm{d} t} \\
& =\sum_{i \in \mathrm{IS}} \sum_{b=q, g} \int_{x_{i}}^{1-\varepsilon} \frac{\mathrm{d} z}{z} \frac{\alpha_{S}(t)}{2 \pi} P_{b a_{i}} \frac{f_{b}\left(x_{i} / z, t\right)}{f_{a_{i}}\left(x_{i}, t\right)} \\
& \quad+\sum_{j \in \mathrm{FS}} \sum_{b=q, g} \int_{\varepsilon}^{1-\varepsilon} \mathrm{d} z \frac{\alpha_{S}(t)}{2 \pi} P_{a_{j} b},
\end{aligned}
$$

where $z \equiv z\left(\Phi_{n+1} / \Phi_{n}\right)$ is an energy-sharing variable and $x$ the momentum fraction of the incoming parton in $\Phi_{n}$. The first term in Eq. (2) corresponds to evolution by initial-state radiation, while the second term represents final-state radiation. Backward evolution [39] for initial-state radiation introduces a ratio of parton distribution functions (PDFs) $f$ in the first term. The quality of the shower real-radiation pattern is governed by the unregularized, dimensionful splitting kernels $P_{i j} \equiv P_{i j}\left(\Phi_{n+1} / \Phi_{n}\right) .{ }^{1}$ For brevity, we will suppress the indices of the splitting functions. The shower will produce an accurate real-emission pattern if the sum of all products of

$\overline{1 \text { We define } P_{i j}}\left(\Phi_{n+1} / \Phi_{n}\right)$ as dimensionful to follow the convention used in the antenna literature $[42,43]$. Thus, $P_{i j}$ corresponds to $\hat{P}_{i j} / t$ in the notation of [41], leading to a marginally different notation compared to the latter. 
splitting probabilities and transition probabilities $\left|\mathcal{M}\left(\Phi_{n}\right)\right|^{2}$ is a good approximation of the full real-emission probability $\left|\mathcal{M}\left(\Phi_{n+1}\right)\right|^{2}$. For a transition from an $n$-particle to an $(n+1)$-particle state, this can be achieved by the (symbolic) replacement

$$
\begin{aligned}
& {\left[\sum_{\Phi_{n}} P\left(\Phi_{n+1} / \Phi_{n}\right)\left|\mathcal{M}\left(\Phi_{n}\right)\right|^{2}\right]} \\
& \quad \rightarrow\left[\sum_{\Phi_{n}} P\left(\Phi_{n+1} / \Phi_{n}\right)\left|\mathcal{M}\left(\Phi_{n}\right)\right|^{2}\right] \\
& \quad \times \frac{\left|\mathcal{M}\left(\Phi_{n+1}\right)\right|^{2}}{\left(\sum_{\Phi_{n}^{\prime}} P\left(\Phi_{n+1} / \Phi_{n}^{\prime}\right)\left|\mathcal{M}\left(\Phi_{n}^{\prime}\right)\right|^{2}\right)} \\
& =\sum_{\Phi_{n}}\left[P\left(\Phi_{n+1} / \Phi_{n}\right)\left|\mathcal{M}\left(\Phi_{n}\right)\right|^{2} \mathcal{R}\left(\Phi_{n+1}\right)\right]
\end{aligned}
$$

Such a process- and multiplicity-dependent redefinition of the splitting kernel is called matrix-element correction (MEC). It is worth noting that this replacement changes both the shower no-emission probability and the real-emission pattern. The real-emission pattern is corrected to a target fixed-order accuracy. However, the accuracy of the partonshower resummation of virtual corrections into Sudakov factors is not improved.

The impact of ME corrections is largest for hard, wellseparated jets, as splitting kernels do not approximate the full fixed-order matrix element well for configurations with hard, well-separated jets. Thus, the most significant improvement of ME corrections can be obtained when correcting the $n$ hardest splittings in the shower cascade. In practise, this means that hardness-ordered parton showers allow for simpler MEC schemes [44-46], which in particular do not require knowledge of high-multiplicity matrix elements as a function of evolution variables. ${ }^{2}$ Instead, it is sufficient that the parton shower generates complete, physical intermediate momenta $\Phi_{n}$ that can be used to evaluate $\left|\mathcal{M}\left(\Phi_{n}\right)\right|^{2}$ numerically. Thus, we will limit our discussion to hardness-ordered shower programs. This will allow for a level of processindependence, and make the iteration of ME corrections possible.

The key technical difficulty for a consistent application of ME corrections is the construction of the sum over partonshower paths in the denominator of the correction factor $\mathcal{R}\left(\Phi_{n+1}\right)$. Since parton showers are formulated as Markov

\footnotetext{
${ }^{2}$ A scheme to correct the hardest emission in angular-ordered showers has been discussed in [47]. This scheme requires to apply the same correction repeatedly, to guarantee that the single hardest emission is corrected to leading-order accuracy. Although promising from the resummation standpoint, it is, however, not obvious how this scheme could be used to correct the $n$ hardest emissions.
}

processes, neither the weight nor the existence of each term in the sum is known a priori when the splitting governed by $P\left(\Phi_{n+1} / \Phi_{n}\right)$ is generated, and all terms have to be reconstructed explicitly.

\section{Matrix-element corrections for ordered parton showers}

The formalism of ME corrections for ordered parton showers (MOPS) is close in spirit to the idea of the iterative MEC approach of $[37,38] .^{3}$ These previous ideas rely on a historyindependent parton shower that is able to fill the complete available phase space. This necessitates abandoning partonshower ordering, i.e. the property that ensures the resummation of large logarithms in ratios of evolution scales. Sensible resummation properties then rely on the introduction of auxiliary functions. Furthermore, configurations with hard well-separated jets might contain poorly understood higherorder corrections. It is thus sensible to limit ME corrections for the parton shower to phase-space regions reachable by an ordered sequence of branchings. This means that we need to rederive appropriate MEC factors $\mathcal{R}\left(\Phi_{n}\right)$ that correctly encode the presence of complicated phase-space constraints due to ordering - making the resulting method substantially different from previous attempts.

To not overcomplicate the derivation of the MOPS formalism, we drop all coupling- and PDF factors in this section. These pieces are evaluated exactly as in an uncorrected parton shower (the probability of a splitting at evolution scale $t$ includes a factor $\alpha_{s}(t) / 2 \pi$, splittings involving initial legs induce ratios of PDFs $f\left(\frac{x}{z}, t\right) / f(x, t)$, cf. Eq. (2)), and do not enter in the MEC factors. Similarly, Sudakov factors are not explicitly written out when demonstrating the MOPS method. The MOPS procedure is applied during the Sudakov veto-algorithm as a redefinition of the splitting kernels, meaning that both the (real) emission probability and the no-emission probabilities are ME corrected. This ensures the unitarity of the method, i.e. that corrections to higher parton multiplicities vanish in observables that are only sensitive to a lower multiplicity.

Consider an arbitrary Born process with factorization scale $t_{\text {fac }} \equiv t\left(\Phi_{0}\right)$ as starting point of the parton shower. The weight of the first branching is

$$
P\left(\Phi_{1} / \Phi_{0}\right) \Theta\left(t\left(\Phi_{0}\right)-t\left(\Phi_{1} / \Phi_{0}\right)\right)\left|\mathcal{M}\left(\Phi_{0}\right)\right|^{2} \mathrm{~d} \Phi_{1},
$$

where the shower is restricted to scales below the factorization scale. For processes that require regularizing cuts at Born level, the matrix element $\left|\mathcal{M}\left(\Phi_{0}\right)\right|^{2}$ can be suitable redefined to include the necessary $\Theta$-functions. To correct the weight

\footnotetext{
${ }^{3}$ A short review of the GKS approach is given in Appendix A.
} 
of the phase-space point $\Phi_{1}$ to the full fixed-order matrix element, all possible emissions from "underlying" Born configurations $\Phi_{0}^{\prime}$ that could have produced the phase-space point $\Phi_{1}$ that we want to correct have to be taken into account. A suitable multiplicative correction factor is thus

$$
\begin{aligned}
\mathcal{R} & \left(\Phi_{1}\right) \\
& =\frac{\left|\mathcal{M}\left(\Phi_{1}\right)\right|^{2}}{\sum_{\Phi_{0}^{\prime}} P\left(\Phi_{1} / \Phi_{0}^{\prime}\right) \Theta\left(t\left(\Phi_{0}^{\prime}\right)-t\left(\Phi_{1} / \Phi_{0}^{\prime}\right)\right)\left|\mathcal{M}\left(\Phi_{0}^{\prime}\right)\right|^{2}} .
\end{aligned}
$$

Applying this correction to each individual splitting and summing over all shower contributions cancels the denominator of Eq. (5) and gives

$$
\begin{aligned}
& \mathcal{R}\left(\Phi_{1}\right) \sum_{\Phi_{0}} P\left(\Phi_{1} / \Phi_{0}\right) \Theta\left(t\left(\Phi_{0}\right)-t\left(\Phi_{1} / \Phi_{0}\right)\right)\left|\mathcal{M}\left(\Phi_{0}\right)\right|^{2} \\
& \quad=\left|\mathcal{M}\left(\Phi_{1}\right)\right|^{2} .
\end{aligned}
$$

The calculation of the correction factor for the weight of a second branching becomes more cumbersome,

$$
\begin{aligned}
\mathcal{R}_{2} & =\frac{\mathcal{M}_{2}}{P_{2}^{1} \mathcal{R}_{1}^{1}\left(P_{1}^{1} \mathcal{M}_{0}^{1}+P_{1}^{2} \mathcal{M}_{0}^{2}\right)+P_{2}^{2} \mathcal{R}_{1}^{2}\left(P_{1}^{3} \mathcal{M}_{0}^{3}+P_{1}^{4} \mathcal{M}_{0}^{4}\right)+P_{2}^{3} \mathcal{M}_{1}^{3}} \\
& =\frac{\mathcal{M}_{2}}{P_{2}^{1} \mathcal{M}_{1}^{1}+P_{2}^{2} \mathcal{M}_{1}^{2}+P_{2}^{3} \mathcal{M}_{1}^{3}} .
\end{aligned}
$$

Since all paths contribute, the nesting of the MOPS factors cancels and the denominator reduces to the sum of the splitting kernels, multiplied with the +1 -particle matrix elements.

Some paths in Fig. 1b are unordered, which leads to +1 MOPS factors of

$\mathcal{R}_{1}^{1}=\frac{\mathcal{M}_{1}^{1}}{P_{1}^{1} \mathcal{M}_{0}^{1}} \quad$ and $\quad \mathcal{R}_{1}^{2}=\frac{\mathcal{M}_{1}^{2}}{P_{1}^{3} \mathcal{M}_{0}^{3}+P_{1}^{4} \mathcal{M}_{0}^{4}}$.

Only one path (brown) contributes to the denominator of $\mathcal{R}_{1}^{1}$ - the other path (red) is unordered.

The correction to the +2 -particle state is

$$
\begin{aligned}
\mathcal{R}_{2} & =\frac{\mathcal{M}_{2}}{P_{2}^{1} \mathcal{R}_{1}^{1} P_{1}^{1} \mathcal{M}_{0}^{1}+P_{2}^{2} \mathcal{R}_{1}^{2} P_{1}^{4} \mathcal{M}_{0}^{4}} \\
& =\frac{\mathcal{M}_{2}}{P_{2}^{1} \mathcal{M}_{1}^{1}+P_{2}^{2} \frac{\mathcal{M}_{1}^{2}}{P_{1}^{3} \mathcal{M}_{0}^{3}+P_{1}^{4} \mathcal{M}_{0}^{4}} P_{1}^{4} \mathcal{M}_{0}^{4}} .
\end{aligned}
$$

$$
\mathcal{R}\left(\Phi_{2}\right)=\frac{\left|\mathcal{M}\left(\Phi_{2}\right)\right|^{2}}{\sum_{\Phi_{1}^{\prime}} P\left(\Phi_{2} / \Phi_{1}^{\prime}\right) \mathcal{R}\left(\Phi_{1}^{\prime}\right) \sum_{\Phi_{0}^{\prime}} \Theta\left(t\left(\Phi_{1}^{\prime} / \Phi_{0}^{\prime}\right)-t\left(\Phi_{2} / \Phi_{1}^{\prime}\right)\right) P\left(\Phi_{1}^{\prime} / \Phi_{0}^{\prime}\right) \Theta\left(t\left(\Phi_{0}^{\prime}\right)-t\left(\Phi_{1}^{\prime} / \Phi_{0}^{\prime}\right)\right)\left|\mathcal{M}\left(\Phi_{0}^{\prime}\right)\right|^{2}} .
$$

Here, the denominator sums over all possible ways how the shower can populate the phase-space point $\Phi_{2}$, taking into account the allowed (ordered) paths through the $\Theta$-functions with the ME corrected parton-shower weights of the intermediate +1 -particle phase-space points. Consequently, $\mathcal{R}\left(\Phi_{2}\right)$ includes the correction factors of the previous order, $\mathcal{R}\left(\Phi_{1}^{\prime}\right)$.

It is useful to illustrate how this relatively complicated recursive definition is obtained with an example. Consider the case of a +2 -particle state shown in Fig. 1. The +2 -particle state on top of the pyramid can be reached from the base of the pyramid by several splitting sequences or "paths". The paths are not necessarily physical but rather serve the purpose of illustration. In Fig. 1a all paths directly contribute to the +2particle state, as each path from the base to the top follows a decreasing (i.e. ordered) sequence of branchings scales. With the shorthands introduced in the caption of Fig. 1 the correction factors for the +1 -particle states are

$\mathcal{R}_{1}^{1}=\frac{\mathcal{M}_{1}^{1}}{P_{1}^{1} \mathcal{M}_{0}^{1}+P_{1}^{2} \mathcal{M}_{0}^{2}} \quad$ and $\quad \mathcal{R}_{1}^{2}=\frac{\mathcal{M}_{1}^{2}}{P_{1}^{3} \mathcal{M}_{0}^{3}+P_{1}^{4} \mathcal{M}_{0}^{4}}$

Both factors contribute to the correction to the +2 -particle state,
The red path in Fig. 1b does not contribute at all to the +2 -particle state since the first branching scale is exceeding the factorization scale, $t_{1}^{2}>t_{\mathrm{fac}}^{2}$. This leads to a cancellation in the first term of the denominator in Eq. (11). The green path is not contributing directly to the +2 -particle state, as $t_{2}^{2}>t_{1}^{3}$. However, since $t_{1}^{3}<t_{\text {fac }}^{3}$, the path is present in $\mathcal{R}_{1}^{2}$. Therefore, the MOPS factor for the +2-particle state depends on more than one "layer" in the paths, and can thus not be calculated by reconstructing only +1 -particle states from the +2 -particle state that should be corrected.

This example highlights the core features of the MOPS method. The recursive structure of the correction factor represents a crucial difference to the GKS method (see Appendix A). At first sight, it seems counter-intuitive that knowledge of ordered and unordered paths is required to correctly calculate the correction factor for a phase-space point that has been generated by an ordered sequence of splittings. However, the necessity becomes clear when the weight of intermediate states is taken into account.

To obtain a uniform accuracy over the complete $n$-parton phase space, states beyond the reach of the parton shower have to be included. We discuss the treatment of these nonshower states in the next section, and present the general formula for the MOPS factor in Sect. 5. 


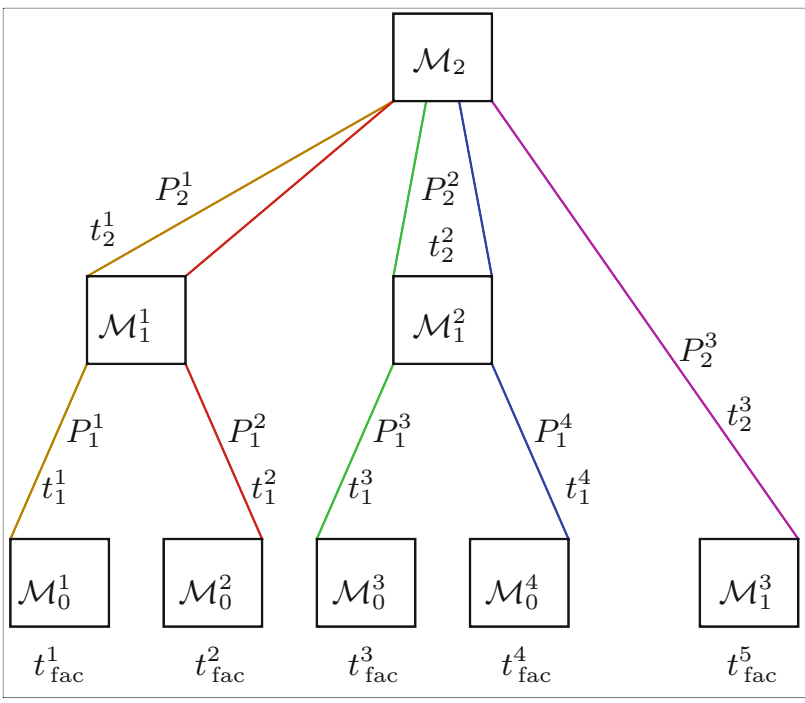

(a) All paths are contributing to the state $\mathcal{M}_{2}$, i.e. all scales fulfill $t_{2}^{i}<t_{1}^{j}<t_{\text {fac }}^{j}$ along the lines.

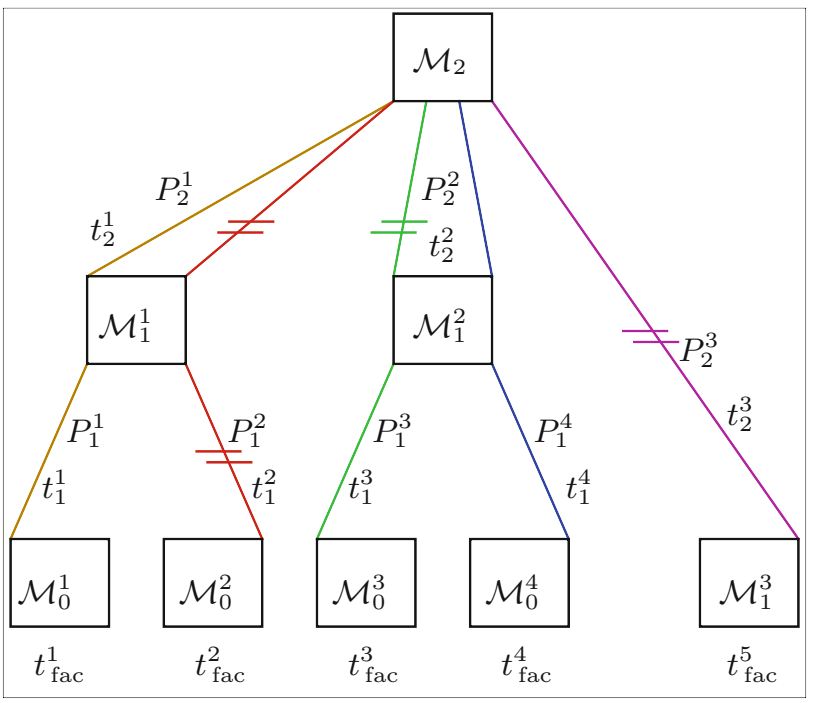

(b) The red and purple paths do not contribute, and the green path only contributes indirectly to the state $\mathcal{M}_{2}$.

Fig. 1 History pyramid to illustrate different levels of contribution to the MOPS factor. The superscripts are numbering the different nodes. We use the shorthands $\mathcal{M}_{X} \equiv\left|\mathcal{M}\left(\Phi_{X}\right)\right|^{2}, t_{X} \equiv t\left(\Phi_{X} / \Phi_{X-1}\right)$, and $P_{X} \equiv P\left(\Phi_{X} / \Phi_{X-1}\right)$. The top layer is the main +2 -particle state and the lower boxes represent the clustered states after one and two successive clusterings, respectively. The scales and splitting probabilities associated with the clusterings are noted along the lines. For illustrative purposes we included a path where the Born state is reached after one clustering (purple line), as present e.g. when combining QCD and electroweak clusterings

\section{Completing the calculation with non-shower configurations}

The MOPS formalism discussed in Sect. 3 only covers the parton-shower phase space characterized by an ordered sequence of splitting scales $\left(t_{\mathrm{fac}}>t_{1}>t_{2} \ldots\right)$. As a consequence, a prescription for the missing phase space is required. The precise definition of these regions depends on the parton shower itself, the starting scale, definition of the evolution variable, and recoil strategy. Configurations can either be forbidden by restricting the first emission to scales below the factorization scale, cuts on lowest-multiplicity phase space points, or by the ordering property of the shower.

When combining non-shower and shower states, care has to be taken to avoid double- or under-counting. As discussed in Sect. 3, the shower off lowest-multiplicity events is treated without any restrictions apart from ordering emissions in the parton-shower evolution variable. Only those highermultiplicity states that cannot be reproduced by showering lower-multiplicity states need to be added explicitly. This criterion supersedes algorithms that rely on the introduction of a merging cut. ${ }^{4}$ Uniform (leading-order) accuracy then is obtained across the complete emission phase space by also

\footnotetext{
4 An arbitrary shower will not correctly describe all sub-leading logs in its evolution variable, so that non-shower configurations may still contain (sub-leading) logarithmic divergences. One famous example of such configurations are the unordered, balancing soft-gluon emissions leading to Parisi-Petronzio scaling in $p_{\perp}$ distributions $[48,49]$.
}

applying a ME corrected shower when adding soft-collinear shower radiation to non-shower states. This will, if performed naively, introduce overlap between (the shower off) different non-shower states. Three steps are required to avoid the overlap:

1. Non-shower events are defined as unordered if no ordered path exists, i.e. if different paths to the same ME state are present, the event is only considered unordered if none of the paths can be reproduced with an ordered sequence of branchings scales.

2. Potential overlap between non-shower states with different parton multiplicities has to be removed, e.g. a maximally unordered +2 -particle state may also be produced as a shower emission off a maximally unordered +1 particle state. The explanation how this overlap is identified and removed in the higher-multiplicity states, is deferred to the end of Sect. 5, since it is helpful to first discuss how non-shower states are showered.

3. States produced by ordered parton showers overlap with soft-collinear radiation attached to non-shower states if the "history" of a phase-space point contains both ordered and unordered paths. Therefore, both have to be ME cor-

\section{Footnote 4 continued}

To avoid such divergences in practice, we only include non-shower phase-space points if each scale at which partons could be recombined (as defined by the shower evolution variable) is above the parton-shower cut-off $t_{\text {cut }} \approx 1 \mathrm{GeV}$. 
rected with correction factors taking into account both possibilities of population.

We now turn to the scale setting in non-shower events with two or more additional partons. From a parton-shower standpoint, there is no a priori guideline how non-shower configurations should be treated. However, since non-shower configurations easily dominate LHC observables depending on many well-separated jets, finding a sensible scale-setting prescription for arbitrary processes will greatly improve the ability of fixed-order + parton-shower calculations to describe data. Variations around the central scale can then be used to assess the precision of the calculation.

To obtain a flexible scale-setting prescription, we borrow the idea of constructing all possible event histories from the CKKW-L [26-28] The aim of the procedure is twofold: define dynamical scales by exploiting the information about the phase-space points with the help of the weight and "substructure" of multi-jet matrix elements, while further ensuring a smooth inclusion of non-shower states with showeraccessible events.

For a sensible scale-setting prescription for non-shower states, we follow an argument similar to the derivation of the MOPS factor. However, ordering considerations should not be applied to non-shower states. Assume that a phasespace point $\Phi_{n+1}$ can be reached from multiple $\Phi_{n}^{\prime}$ states by splitting an external leg. The contribution to the cross section due to splitting a single leg can be approximated by

$\alpha_{s}\left(t\left(\Phi_{n+1} / \Phi_{n}^{\prime}\right)\right) P\left(\Phi_{n+1} / \Phi_{n}^{\prime}\right) \alpha_{s}^{n}\left(t_{n}^{\mathrm{eff}}\right)\left|\mathcal{M}\left(\Phi_{n}^{\prime}\right)\right|^{2}$,

where $t_{n}^{\text {eff }}$ is a suitable scale for the "underlying" $n$-particle state. To obtain the correct (leading-order) result when summing over all possible splittings $\Phi_{n}^{\prime} \rightarrow \Phi_{n+1}$, we can apply the corrective factor

$\mathcal{R}\left(\Phi_{n+1}\right)=\frac{\alpha_{s}^{n+1}\left(t_{n+1}^{\text {eff }}\right)\left|\mathcal{M}\left(\Phi_{n+1}\right)\right|^{2}}{\sum_{\Phi_{n}^{\prime}} \alpha_{s}\left(t\left(\Phi_{n+1} / \Phi_{n}^{\prime}\right)\right) P\left(\Phi_{n+1} / \Phi_{n}^{\prime}\right) \alpha_{s}^{n}\left(t_{n}^{\text {eff }}\right)\left|\mathcal{M}\left(\Phi_{n}^{\prime}\right)\right|^{2}}$,

where $t_{n+1}^{\text {eff }}$ is the desired (currently unknown) scale for the $(n+1)$-particle state. To find a suitable scale, note that

(a) if one splitting dominates over all other splittings, then a natural scale to capture the dynamics is strongly correlated with the relative jet separation of the dominant splitting,

(b) if no splitting dominates, i.e. all splittings contribute democratically, there should be no strong preference for a scale, and a weighted average of jet separations seems appropriate.
Leaving aside the complications (and bias) induced by ordering constraints, an identical argument holds for partonshower-produced states. In this case, the requirements above are fulfilled by keeping the characteristic shower-induced $\alpha_{s}$ factors for every ME corrected shower splitting. This would be guaranteed if the $\alpha_{s}$ factors in Eq. (13) would be identified by

$$
\begin{aligned}
& \alpha_{s}^{n+1}\left(t_{n+1}^{\mathrm{eff}}\right) \\
& =\frac{\sum_{\Phi_{n}^{\prime}} \alpha_{s}\left(t\left(\Phi_{n+1} / \Phi_{n}^{\prime}\right)\right) P\left(\Phi_{n+1} / \Phi_{n}^{\prime}\right) \alpha_{s}^{n}\left(t_{n}^{\mathrm{eff}}\right)\left|\mathcal{M}\left(\Phi_{n}^{\prime}\right)\right|^{2}}{\sum_{\Phi_{n}^{\prime}} P\left(\Phi_{n+1} / \Phi_{n}^{\prime}\right)\left|\mathcal{M}\left(\Phi_{n}^{\prime}\right)\right|^{2}},
\end{aligned}
$$

since then Eq. (13) is a simplified MEC factor. For ordered parton-shower sequences, Eq. (14) will not lead to the correct result. It is, however, well-suited as a scale-setting prescription for non-shower configurations. We will use Eq. (14) as the definition of the effective scales below, i.e. we set the renormalization and factorization scales for non-shower events to $t$ eff . The effective scale also serves as a shower (re)starting scale. The variation of the effective scale may act as an uncertainty estimate of the prescription.

An expression for the effective scale could also have been obtained by including PDF ratios in Eq. (13), which would mean that the choice of effective scale captured dynamics of underlying "hadronic" cross sections. We do not implement such a scale-setting prescription since we believe that the scale setting should be based on perturbative parton-level quantities.

Note that the scale-setting mechanism in Eq. (14) allows for $t_{\text {fac }}<t$ eff if the scales entering the calculation are sufficiently large. An example of such a configuration are nonshower states with multiple hard (and possibly balancing) jets without $p_{\perp}$ hierarchy. In this case, using a scale defined for the lowest-multiplicity process can result in pathologies [50]. It is desirable that $t^{\text {eff }}$ is not bounded by $t_{\text {fac }}$, the factorization scale assigned to a fictitious lowest-multiplicity process. Instead, $t$ eff should provide a more "natural" scale for this genuine multi-jet configuration. Furthermore, $t^{\text {eff }}$ is bound to remain in the perturbative region, since we only include non-shower phase space points for which clustering scales (as defined by the shower evolution variable) are above the parton-shower cut-off.

In Sect. 6 we will show that the scale setting outlined in this section results in a very good description of LHC data.

\section{The complete algorithm}

In this section, we summarize the combined fixed-order + parton-shower algorithm, and present the general form of the MOPS factor. The scheme introduces ME correction for 
several ordered consecutive parton-shower emissions. This is in general obtained by applying the MOPS factor result for small $\vec{p}_{\perp}$ of the combined Born $+n$-parton system. If instead a hierarchy $t_{\mathrm{fac}}>t_{n-1}^{\text {eff }}>t_{n}$ can be constructed,

$$
\begin{aligned}
\mathcal{R}\left(\Phi_{n+1}\right)=\left|\mathcal{M}\left(\Phi_{n+1}\right)\right|^{2} & {\left[\sum_{\Phi_{n}^{\prime}} P\left(\Phi_{n+1} / \Phi_{n}^{\prime}\right) \mathcal{R}\left(\Phi_{n}^{\prime}\right) \sum_{\Phi_{n-1}^{\prime}} \Theta\left(t\left(\Phi_{n}^{\prime} / \Phi_{n-1}^{\prime}\right)-t\left(\Phi_{n+1} / \Phi_{n}^{\prime}\right)\right) P\left(\Phi_{n}^{\prime} / \Phi_{n-1}^{\prime}\right) \mathcal{R}\left(\Phi_{n-1}^{\prime}\right)\right.} \\
\times & \prod_{k=n-2}^{k \leq 1}\left(\sum_{\Phi_{k}^{\prime}} \Theta\left(t\left(\Phi_{k+1}^{\prime} / \Phi_{k}^{\prime}\right)-t\left(\Phi_{k+2}^{\prime} / \Phi_{k+1}^{\prime}\right)\right) P\left(\Phi_{k+1}^{\prime} / \Phi_{k}^{\prime}\right) \mathcal{R}\left(\Phi_{k}\right)\right) \\
& \left.\sum_{\Phi_{0}^{\prime}} \Theta\left(t\left(\Phi_{1}^{\prime} / \Phi_{0}^{\prime}\right)-t\left(\Phi_{2}^{\prime} / \Phi_{1}^{\prime}\right)\right) P\left(\Phi_{1}^{\prime} / \Phi_{0}^{\prime}\right) \Theta\left(t\left(\Phi_{0}^{\prime}\right)-t\left(\Phi_{1}^{\prime} / \Phi_{0}^{\prime}\right)\right)\left|\mathcal{M}\left(\Phi_{0}^{\prime}\right)\right|^{2}\right]^{-1}
\end{aligned}
$$

to the splitting kernel. When including the correct weight of each possible path, the result exhibits a recursive structure, where $\mathcal{R}\left(\Phi_{n+1}\right)$ includes the correction factors of all previous orders, $\mathcal{R}\left(\Phi_{n}^{\prime}\right)$ to $\mathcal{R}\left(\Phi_{1}^{\prime}\right)$. Once non-shower states are added, their contributions to the MOPS factor are taken into account as well.

Non-shower states are added as new configurations, with renormalization and factorization scales calculated through

$\alpha_{s}^{n+1}\left(t_{n+1}^{\text {eff }}\right)=\frac{\sum_{\Phi_{n}^{\prime}} \alpha_{s}\left(t\left(\Phi_{n+1} / \Phi_{n}^{\prime}\right)\right) P\left(\Phi_{n+1} / \Phi_{n}^{\prime}\right) \alpha_{s}^{n}\left(t_{n}^{\text {eff }}\right)\left|\mathcal{M}\left(\Phi_{n}^{\prime}\right)\right|^{2}}{\sum_{\Phi_{n}^{\prime}} P\left(\Phi_{n+1} / \Phi_{n}^{\prime}\right)\left|\mathcal{M}\left(\Phi_{n}^{\prime}\right)\right|^{2}}$.

This should ensure that the dynamics of the process are encoded in a sensible scale choice, without the scalesetting prescription being based on process- or multiplicitydependent arguments.

Since non-shower states are included without a hard cutoff (e.g. a merging scale), the effective scale $t^{\text {eff }}$ may differ significantly from the factorization scale $t_{\text {fac }}$. In this case, we further attach Sudakov factors by means of trial showering $[26,27]$ to the non-shower states to include a sensible suppression due to the resummation of large logarithms of $t_{\mathrm{fac}} / t^{\text {eff }}$. This is relatively straight-forward for +2 -particle states-a Sudakov factor $\Delta\left(t_{\text {fac }}, t_{2}^{\text {eff }}\right)$ is applied to ensure a sensible result if the $\vec{p}_{\perp}$ of the combined Born +2 -parton system is small. For higher-multiplicity non-shower states, more scale hierarchies arise, and a more detailed scheme is necessary to cover all relevant cases. However, only two types of scale hierarchies can remain after removing the overlap between $n$-particle non-shower events and states that are produced by showering lower-multiplicity non-shower configurations: the ordering $t_{\mathrm{fac}}>t_{n}^{\text {eff }}$, or the ordering $t_{\mathrm{fac}}>t_{n-1}^{\text {eff }}>$ $t_{n} .5$ The hierarchy $t_{\text {fac }}>t_{n}^{\text {eff }}$ is again ameliorated by applying a single Sudakov factor $\Delta\left(t_{\mathrm{fac}}, t_{n}^{\text {eff }}\right)$ to produce a sensible

\footnotetext{
${ }^{5}$ Consider a non-shower (unordered) +4-particle state. After computing effective scales, it is possible that a scale hierarchy $t_{\mathrm{fac}}>t_{2}^{\text {eff }}>$ $t_{3}>t_{4}$ exists. Such a configuration can be obtained in several ways
}

then a product of Sudakov factors $\Delta\left(t_{\mathrm{fac}}, t_{n-1}^{\text {eff }}\right) \Delta\left(t_{n-1}^{\text {eff }}, t_{n}\right)$ is appropriate. This guarantees a uniform weighting of $+n$ particle events arising from either $+n$-particle non-shower states or showered $+(n-1)$-particle configurations. Note that the Sudakov factors $\Delta\left(t_{\mathrm{fac}}, t^{\mathrm{eff}}\right)$ are unity if $t_{\mathrm{fac}}<t^{\text {eff }}$.

The information about the different types of scale hierarchies are also used to remove the overlap between nonshower states with different parton multiplicities. States with scale hierarchies of the type $t_{n-m}^{\text {eff }}>t_{n-(m-1)}>\cdots>t_{n}$ are removed for $m \geq 2$. For states that contain the hierarchy $t_{n-1}^{\text {eff }}>t_{n}$, the event is removed if the clustered $+(n-1)$ particle state is itself an unordered state. Events without scale hierarchies that could have resulted from showering lowermultiplicity states are kept; that includes all +2 -particle states with unordered scales $t_{2}>t_{1}$ and +1 -particle states with $t_{1}>t_{\mathrm{fac}}$. For the interested reader we include further methodological instructions in Appendix C.

\section{Results}

In this section, we present results obtained with the new method, including both the MOPS factor and the non-shower states (called "MOPS + unordered" in the following). A detailed validation can be found in Appendix B. The analyses are performed with RIVET [51]. We begin this section

\footnotetext{
Footnote 5 continued

showering lower-multiplicity non-shower states. (a) If the reconstructed underlying +2-particle state is not shower-like (i.e. unordered), then the +4-particle state with the above hierarchy can be produced by adding two ordered shower emissions to the +2 -particle state. Thus, the state is included by showering a non-shower +2 -particle state. (b) If the reconstructed +2 -particle state can be reached by an ordered sequence of emissions, and furthermore $t_{3}>t_{4}$ then the "unordering" stems from the +2 -particle to +3 -particle transition. Thus, the +4 -particle configuration can be reached by adding one ordered shower emission to a non-shower +3 -particle state. In conclusion, the states with this more complex scale hierarchy should not be included through a non-shower +4-particle input, since this would result in over-counting.
} 
by studying the effect of the new method on jet separations, before moving to comparisons to LHC data. In both cases, we juxtapose the results with the GKS ME corrections implemented in VINCIA. The GKS MECs scheme includes emissions above the factorization scale $t_{\text {fac }}$ (see Appendix A2 for how those are generated) as does the MOPS + unordered method by adding non-shower +1 -particle states. Emissions with scales $t_{1}>t_{\text {fac }}$ would not naturally be present in the pure or MOPS corrected shower, where Born states are showered beginning at $t_{\mathrm{fac}}$. For the following results we add +1 particle states with scales $t_{1}>t_{\text {fac }}$ explicitly to the pure and MOPS corrected shower, and shower these states using $t_{1}$ as shower starting scale. This decreases the significance of including non-shower states w.r.t comparing to a strictly ordered shower evolution, but should avoid using an "overly conservative" shower setup when comparing to default VINCIA.

\subsection{Theory comparisons}

Here, the general features of the new method are illustrated by discussing jet resolution scales. These variables show significant sensitivity to hard, well-separated jets as well as partonshower resummation, and they can thus be used to gauge the effect of different pieces in the calculation. To not obscure the Sudakov shapes of the parton shower at low jet resolution, we do not include multiparton interactions.

Hadron-level results for hadronic $Z$ decays and Drell-Yan events are presented in Fig. 2. The results have the expected behavior: at low jet resolution, parton-shower effects dominate, while non-shower states contribute mainly to large jet scales. Hence, the MOPS factor is dominating the observable at low scales. At LEP, shower states remain a dominant contribution even when modeling well-separated jets, and the effect of non-shower states remains at below $10 \%$ per bin. Results at the LHC are in stark contrast to this. There, the influence of shower configurations decreases substantially for large jet resolution, and non-shower phase-space regions become increasingly important. The uncertainty from varying the effective scale is not significant at LEP, and should thus not be considered a realistic uncertainty estimate. At LHC, the variation of $t^{\text {eff }}\left(=t_{\text {fac }}=t_{\text {ren }}=t_{\text {start }}\right)$ is larger, and increases for high jet resolution, as expected from varying scales in a tree-level fixed-order variation. At low resolution, we observe a small increase in the scale uncertainty, which stems from the interplay of very large $\alpha_{s}$ values with the Sudakov factors that are applied to non-shower states.

By comparing with previous ideas below, we hope to understand the short-comings and benefits of our MOPS + unordered prescription. In Fig. 3 we compare the results of VINCIA2.2 without corrections, with the MOPS correction, MOPS + unordered scheme, and VINCIA2.0.01 with smooth ordering for the GKS ME corrected orders.
The MOPS correction for purely evolution-induced events is small for all jet resolutions. Differences are mostly at the level of $1-5 \%$, illustrating that the uncorrected shower already describes the matrix elements well in phase-space regions reachable by showering. As discussed above, the jet resolution scales exhibit a Sudakov suppression for small values. In the Sudakov region, the corrected predictions should not deviate greatly from the "plain" shower result. This is indeed the case for both the MOPS + unordered and the GKS MECs method for very small resolution scales. The GKS MECs method generates more events with larger $d_{m m+1}$ separation. Due to the unitarity of the shower, this leads to a depletion of events with small separation compared to the pure shower. The behavior is consistent with the findings in [38], where differences between strong and smooth ordering have been investigated. The impact of non-shower states in the MOPS + unordered scheme remains noticeable close to the peak of the distribution, although the modeling of the Sudakov region approaches the uncorrected shower more quickly than for the GKS MECs method. This means that the handling of non-shower states with large scale hierarchies (cf. end of Sect. 5) is important in our approach. Merging approaches commonly discard non-shower states with separation below a certain (merging) scale.

In conclusion, we believe that the MOPS + unordered scheme has desirable features, and that the choices in the method lead to the expected behavior.

\subsection{Comparisons to data}

To assess how the method performs for realistic observables, we now turn to Drell-Yan + jets measurements at the LHC. All curves employ the NNPDF 2.1 LO PDF set [56] and use the corresponding strong coupling $\alpha_{s}\left(k_{\mu} t\right)$ with one-loop running, $\alpha_{s}\left(m_{Z}^{2}\right)=0.13$, and $k_{\mu}=1$ for all branchings. We use these settings to compare all schemes on equal footing and choose $k_{\mu}=1$ as required for the calculation of the effective scale. ${ }^{6}$ Soft-physics parameters are kept at their current VINCIA default values. The default VINCIA2.0.01 tune [38] corresponds to different $\alpha_{s}$ settings. While this results in a slightly better data description, it does not alter the general observations and conclusions of this section.

In Fig. 4 we confront the results of VINCIA2.2 without corrections, with the MOPS correction, MOPS + unordered scheme, and VINCIA2.0.01 with GKS ME corrections with ATLAS [52,53] and CMS [54,55] measurements.

As already seen in Sect. 6.1, the effect of the MOPS correction factor is small for all observables. This is of benefit for the description of the Drell-Yan $p_{\perp}$ spectrum (upper left

\footnotetext{
${ }^{6}$ Different $k_{\mu}$ values for different branching types invalidate the interpretation of the effective scale as a single parton-shower starting scale for subsequent showering.
} 


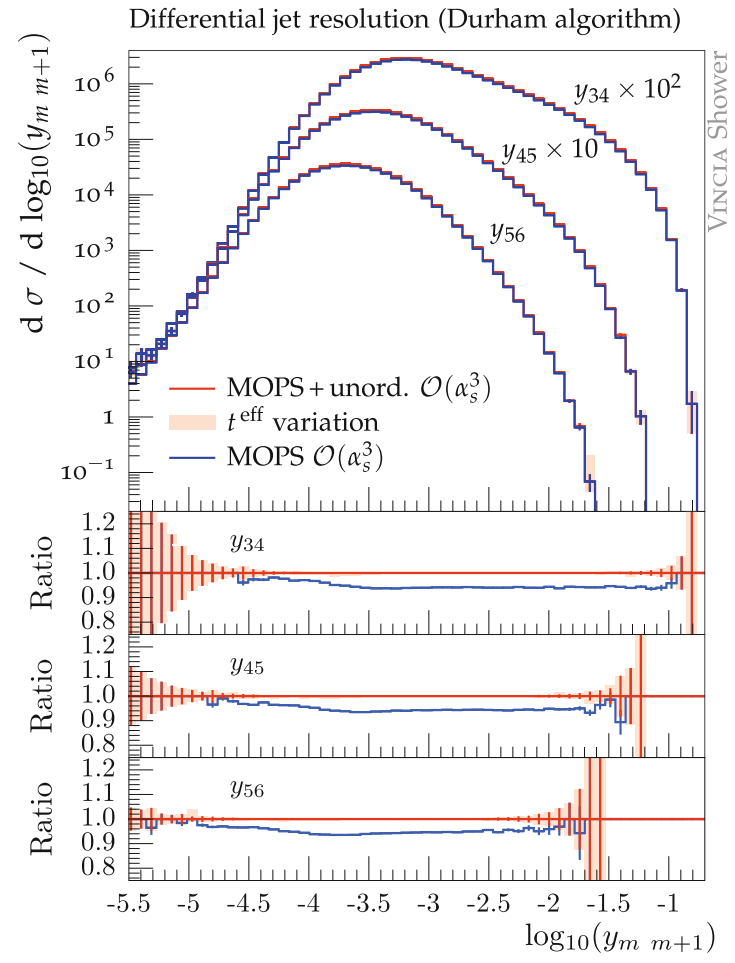

(a) $e^{+} e^{-} \rightarrow Z \rightarrow$ jets $@ 91 \mathrm{Gev}$

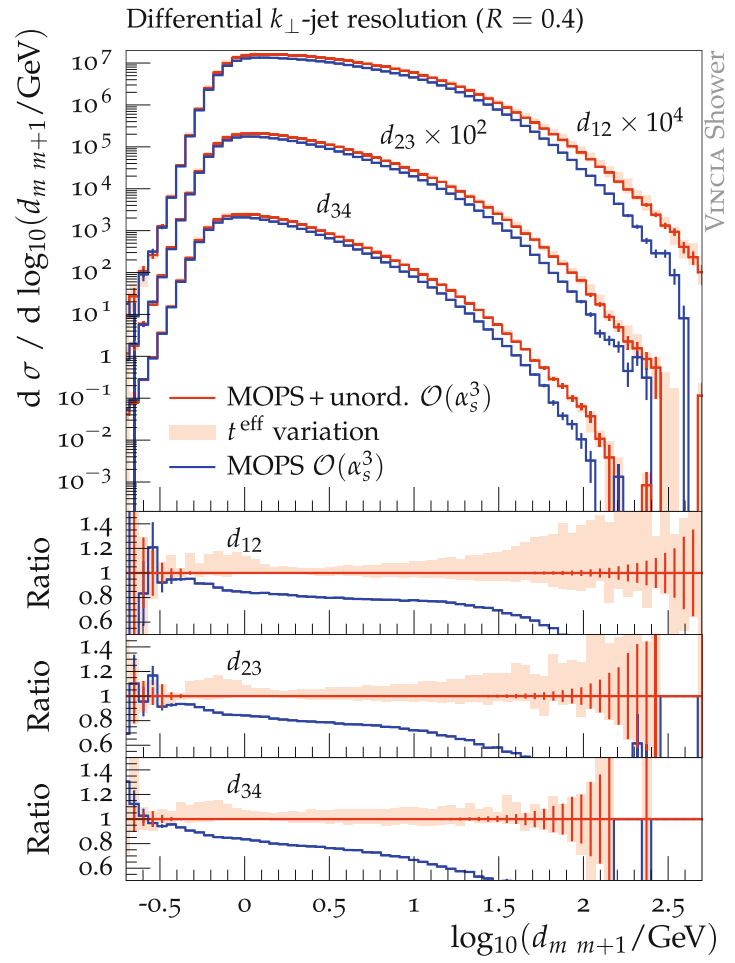

(b) $p p \rightarrow Z+$ jets $@ 7 \mathrm{TeV}$

Fig. 2 PYTHIA8.2.26+VINCIA2.2 predictions for jet resolution measures $d_{m m+1}$ and $y_{m m+1}$ (the longitudinally invariant $k_{\perp}$ jet algorithm with $R=0.4$ for hadronic initial states and the Durham jet algorithm for lepton collisions). ME corrections are applied for $\leq 3$ emissions. The red band is obtained by varying the effective scale $t^{\text {eff }}[\mathrm{GeV}]$ in non-shower events by factors of 2

Differential $k_{\perp}$-jet resolution $(R=0.4)$

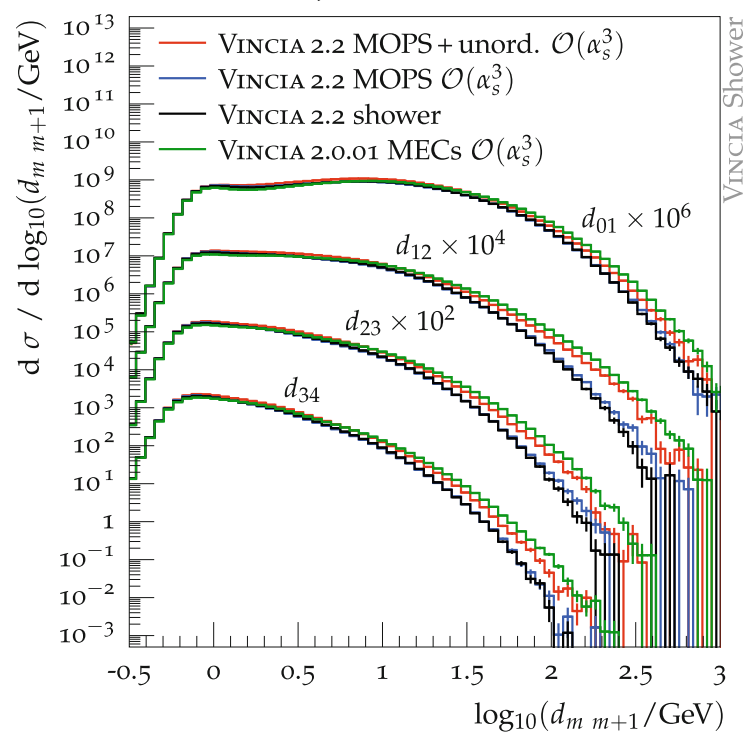

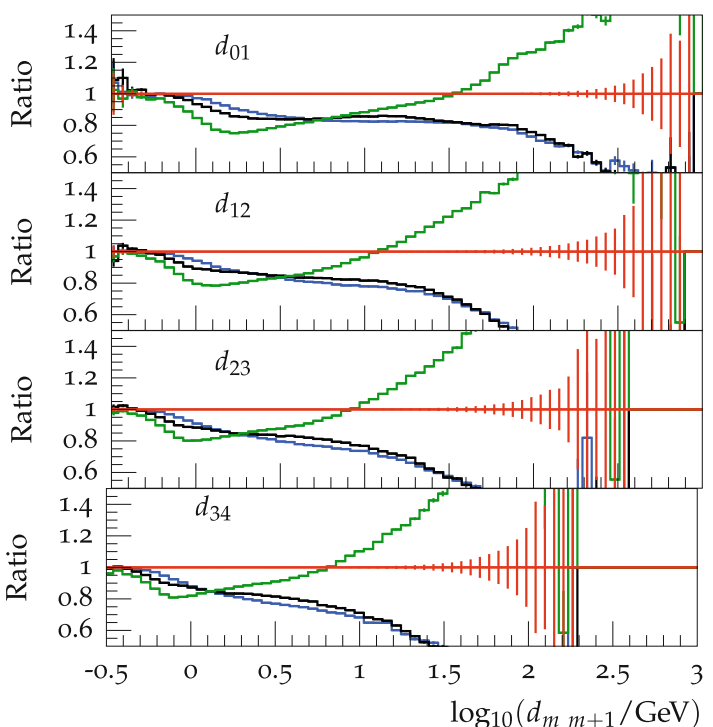

Fig. 3 PYTHIA8.2.26+VinCIA2.2 and PYTHIA8.2.15+VInCIA2.0.01 predictions for jet resolution measures in Drell-Yan events @ 7 TeV. ME corrections are applied for $\leq 3$ emissions

panel of Fig. 4), for which the plain shower already offers a sensible data description. The quality of the description also remains unchanged for the MOPS + unordered scheme. The other observables in Fig. 4 test the existence of hard, well- separated emissions in the tails of the distributions and are thus poorly modeled with the parton shower alone. We find a very good data description with the MOPS + unordered scheme. In particular, the quality of the data description in 
ATLAS, $Z \rightarrow \mu \mu$ "dressed", inclusive, $\sqrt{s}=7 \mathrm{TeV}$

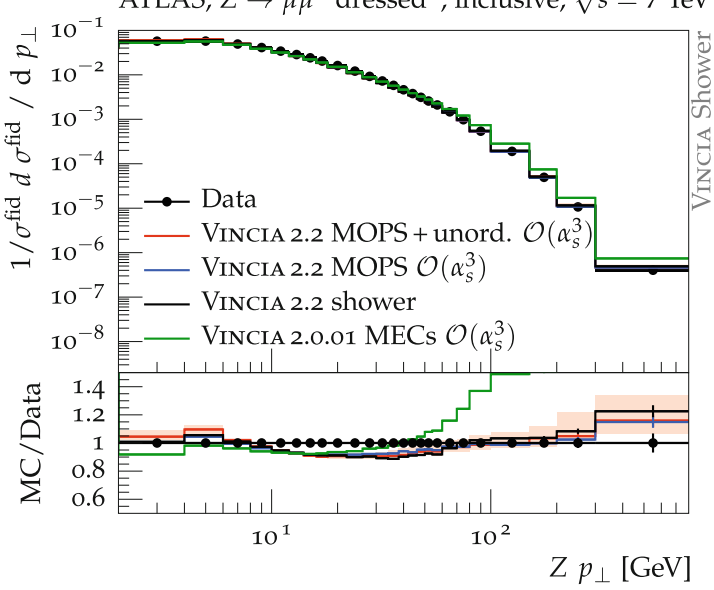

CMS, leading jet $p_{\perp}, \sqrt{s}=7 \mathrm{TeV}$

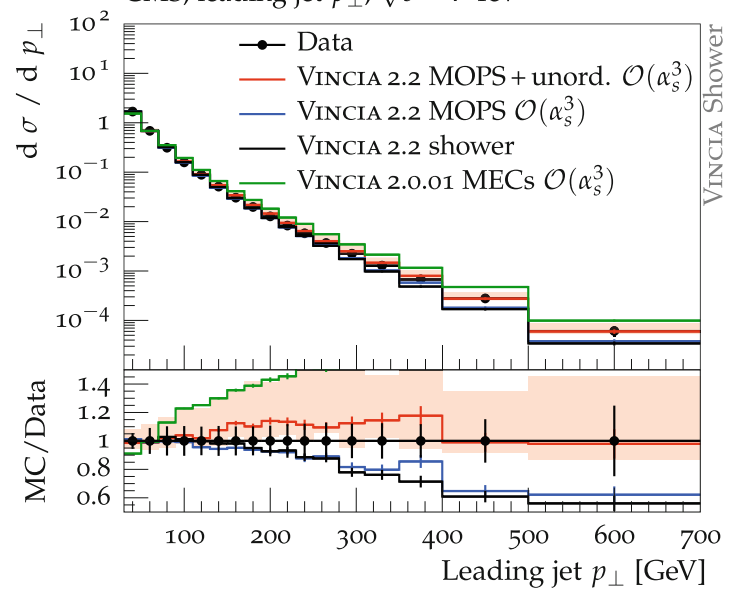

ATLAS, scalar $p_{\perp}$ sum of jets, $\sqrt{s}=7 \mathrm{TeV}$

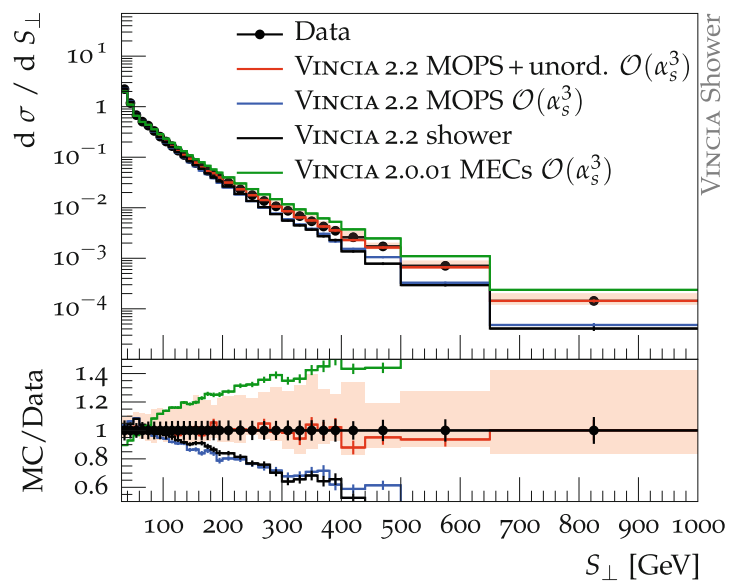

CMS, $\Delta \phi(\mathrm{Z}, \mathrm{J} 1), \sqrt{s}=7 \mathrm{TeV}$

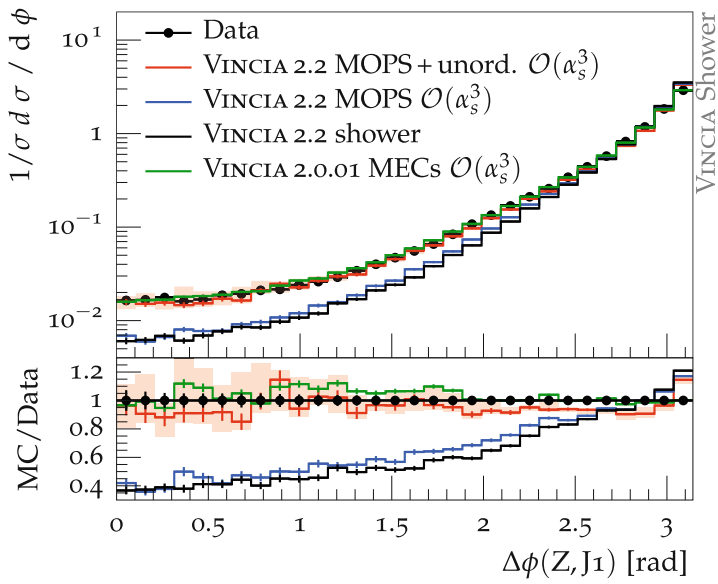

Fig. 4 PYTHIA8.2.26+VinCIA2.2 and PYTHIA8.2.15 + VinCIA2.0.01 predictions compared to ATLAS data from [52,53] and CMS data from [54,55]. RIVET analyses ATLAS_2013_I1230812, ATLAS_2014_I1300647, CMS_2013_I1209721, and CMS_2015_I1310737. For the leading jet $p_{\perp}$ and the scalar $p_{\perp}$ sum of jets the predictions are rescaled to the experimental inclusive one-jet cross section. ME corrections are applied for $\leq 3$ emissions. The red band is obtained by varying the effective scale $t^{\text {eff }}[\mathrm{GeV}]$ in non-shower events by factors of 2

our scheme relies crucially on the treatment of non-shower states. The scale-setting mechanism presented in Sect. 4 produces promising results, with the naive central scale choice close to the data, but with a large, leading-order-like uncertainty due to scale variations. We anticipate that the width of the band will decrease when performing a next-to-leadingorder calculation with a similar scale choice. The uncertainty due to scale variations is largest in phase-space regions most sensitive to non-shower contributions. For the $S_{\perp}$ and leading jet $p_{\perp}$ distributions, the results of the GKS MECs approach touch the uncertainty bands attributed to non-shower events at low values, but are outside of the band in regions influenced by multiple hard jets. Both of these observables are much improved in the MOPS + unordered method, compared to the uncorrected shower. For the angle between the $Z$-boson and the hardest jet we observe a satisfactory data description for both our new method and VINCIA2.0.01.

\section{Conclusions}

We have presented an algorithm to obtain fixed-order accurate predictions combined with all-order parton-shower evolution that produces finite and non-overlapping results without introducing a merging scale. The new algorithm requires the introduction of a sophisticated matrix-element correction method for evolution-induced configurations. States beyond the reach of the parton shower are included with a systematic scale-setting procedure. This smoothly combines nonshower configurations and states produced in the ordered parton-shower evolution. The algorithm does not depend on specific properties of the parton shower and allows for arbitrary dead zones (which may be required by resummation considerations). The new fixed-order + parton-shower scheme has been implemented in the VINCIA parton shower 
and will be made publicly available upon the VINCIA2.2 release.

The effect of including ME corrections for ordered partonshower splittings is minor compared to the uncorrected shower. This means that the method does not deteriorate the shower resummation, and gives us confidence that the improvement does not interfere with other improvement strategies [57-59]. The main improvements stem from a careful treatment of contributions from phase-space regions that are not accessible by ordered parton showers. Such contributions are included with a sophisticated scale-setting prescription. For hadronic initial we find the scale setting to have a sizable influence on observables, since large parts of phase space are not shower accessible. We presented comparisons to data for the $p p \rightarrow Z+$ jets process and found the results of our new algorithm to be in good agreement with the data.

Acknowledgements We would like to thank Stefan Höche and Peter Skands for helpful discussions, and Peter Skands for comments on the manuscript. This work was supported by the Australian Research Council and by Fermi Research Alliance, LLC under Contract No. DE-AC0207CH11359 with the U.S. Department of Energy, Office of Science, Office of High Energy Physics. NF thanks the SLAC and Fermilab theory divisions for hospitality during the course of this work.

Open Access This article is distributed under the terms of the Creative Commons Attribution 4.0 International License (http://creativecomm ons.org/licenses/by/4.0/), which permits unrestricted use, distribution, and reproduction in any medium, provided you give appropriate credit to the original author(s) and the source, provide a link to the Creative Commons license, and indicate if changes were made.

Funded by SCOAP S $^{3}$.

\section{Appendix A: Review of GKS matrix-element corrections}

Iterative ME corrections have first been introduced in [37], and have been applied to colorless resonance decays [37] as well as to initial-state radiation [38]. Finite multiplicative correction factors are applied order by order in perturbation theory as the shower evolves. The MEC factor $\mathcal{R}\left(\Phi_{n+1}\right)$ replaces the splitting kernels by a ratio of tree-level matrix elements. Symbolically, the correction factor can be written as

$$
\begin{aligned}
& P\left(\Phi_{n+1} / \Phi_{n}\right) \longrightarrow \mathcal{R}\left(\Phi_{n+1}\right) P\left(\Phi_{n+1} / \Phi_{n}\right) \\
& \equiv \frac{\left|\mathcal{M}\left(\Phi_{n+1}\right)\right|^{2}}{\sum_{\Phi_{n}^{\prime}} P\left(\Phi_{n+1} / \Phi_{n}^{\prime}\right)\left|\mathcal{M}\left(\Phi_{n}^{\prime}\right)\right|^{2}} P\left(\Phi_{n+1} / \Phi_{n}\right) .
\end{aligned}
$$

The denominator sums over all possible $n$-particle states through which the shower could have produced the $(n+1)$ particle state.
A 1. Smoothly ordered showers

The MEC formalism in [37,38] requires a historyindependent parton shower that covers the full phase space for the ME corrected orders. Therefore, VINCIA introduces the concept of smooth ordering. At any stage of the evolution the following procedure determines at which scale the shower off each parton in a $(n+1)$-particle state is restarted:

- Find all physical clusterings $\Phi_{n+1} \rightarrow \Phi_{n}^{i}$ and their branching scales $t\left(\Phi_{n+1} / \Phi_{n}^{i}\right)$. The reference scale is the minimum of all scales, $\hat{t}\left(\Phi_{n+1}\right)=\min _{i}\left(t\left(\Phi_{n+1} / \Phi_{n}^{i}\right)\right)$.

- Divide the $(n+1)$-particle state into a set of "ordered" and "unordered partons". For more details see [38].

- The evolution of "ordered partons" is restart at the reference scale $\hat{t}$. "Unordered partons" are allowed to radiate up to the phase-space maximum, but with the suppression factor

$$
\begin{gathered}
P_{\text {imp }}\left(\hat{t}\left(\Phi_{n+1}\right), t\left(\Phi_{n+2} / \Phi_{n+1}\right)\right) \\
=\frac{\hat{t}\left(\Phi_{n+1}\right)}{\hat{t}\left(\Phi_{n+1}\right)+t\left(\Phi_{n+2} / \Phi_{n+1}\right)} .
\end{gathered}
$$

When taking smooth ordering into account, the MEC factor (A1) should be defined as

$$
\mathcal{R}\left(\Phi_{n+1}\right)=\frac{\left|\mathcal{M}\left(\Phi_{n+1}\right)\right|^{2}}{\sum_{\Phi_{n}^{\prime}} \mathcal{O}\left(\hat{t}\left(\Phi_{n}^{\prime}\right), t\left(\Phi_{n+1} / \Phi_{n}^{\prime}\right)\right) P\left(\Phi_{n+1} / \Phi_{n}^{\prime}\right)\left|\mathcal{M}\left(\Phi_{n}^{\prime}\right)\right|^{2}} .
$$

The ordering criterion reflects the different treatment of partons,

$$
\begin{aligned}
\mathcal{O} & \left(\hat{t}\left(\Phi_{n}^{\prime}\right), t\left(\Phi_{n+1} / \Phi_{n}^{\prime}\right)\right) \\
& = \begin{cases}P_{\text {imp }}\left(\hat{t}\left(\Phi_{n}^{\prime}\right), t\left(\Phi_{n+1} / \Phi_{n}^{\prime}\right)\right) & \text { for a branching of an "unordered parton", }\end{cases}
\end{aligned}
$$

The procedure guarantees a history-independent parton shower that covers the full kinematic range. However, it introduces complications that are hard to constrain from QCD considerations alone.

\section{a. Sudakov factors in unordered regions}

Consider the exclusive Born+jet cross section at the end of parton shower with the following evolution. The shower starts at the factorization scale of the Born process $t_{\text {fac }}$. After the branching at scale $t_{1}<t_{\text {fac }}$, all partons explore their full kinematic range up to the scale $t_{\max }$ and are evolved down to the shower cut-off $\mu_{\mathrm{c}}$. Dropping the PDF factor for the second leg and suppressing most dependences of the splittings kernels, the exclusive cross section for this evolution sequence reads 


$$
\begin{aligned}
& \mathrm{d} \sigma_{1}\left(\mu_{\mathrm{c}}\right)=\Pi_{1}\left(t_{\max }, \mu_{\mathrm{c}}\right) \cdot \alpha_{s}\left(t_{1}\right) P\left(t_{1}\right) \\
& \quad \times \frac{f_{1}\left(x_{1}, t_{1}\right)}{f_{0}\left(x_{0}, t_{1}\right)} \Pi_{0}\left(t_{\mathrm{fac}}, t_{1}\right) \cdot f_{0}\left(x_{0}, t_{\mathrm{fac}}\right)\left|\mathcal{M}\left(\Phi_{0}\right)\right|^{2} \mathrm{~d} \Phi_{1} .
\end{aligned}
$$

The no-emission probability $\Pi_{1}\left(t_{\max }, \mu_{\mathrm{c}}\right)$ can be split up into an ordered part $\Pi_{1}\left(t_{1}, \mu_{\mathrm{c}}\right)$ and a part that reflects the evolution in the unordered region $\Pi_{1}^{\mathrm{uo}}\left(t_{\max }, t_{1}\right)$. We use the relation [60]

$\Pi_{n}\left(t_{n}, t_{n+1}\right)=\frac{f_{n}\left(x_{n}, t_{n+1}\right)}{f_{n}\left(x_{n}, t_{n}\right)} \Delta_{n}\left(t_{n}, t_{n+1}\right)$

to write the cross section in terms of Sudakov factors,

$$
\begin{aligned}
& \mathrm{d} \sigma_{1}\left(\mu_{\mathrm{c}}\right)=\Pi_{1}^{\mathrm{uo}}\left(t_{\max }, t_{1}\right) \cdot f_{1}\left(x_{1}, \mu_{\mathrm{c}}\right) \Delta_{1}\left(t_{1}, \mu_{\mathrm{c}}\right) \\
& \quad \times \alpha_{s}\left(t_{1}\right) P\left(t_{1}\right) \Delta_{0}\left(t_{\mathrm{fac}}, t_{1}\right) \cdot\left|\mathcal{M}\left(\Phi_{0}\right)\right|^{2} \mathrm{~d} \Phi_{1} .
\end{aligned}
$$

The no-emission probability $\Pi_{1}^{\mathrm{uo}}\left(t_{\max }, t_{1}\right)$ remains in the cross section. In VINCIA this factor is defined as

$$
\begin{aligned}
& \Pi_{1}^{\mathrm{uo}}\left(t_{\max }, t_{1}\right) \\
& \quad=\exp \left(-\sum_{1 \rightarrow 2} \int \mathrm{d} z \int_{t_{1}}^{t_{\max }} \mathrm{d} t \frac{f_{2}\left(x_{2}, t_{1}\right)}{f_{1}\left(x_{1}, t_{1}\right)} \alpha_{S}(t) P_{\text {imp }} P(t, z)\right) .
\end{aligned}
$$

Here, the scale in the PDF ratio is fixed to the scale of the previous emission to ensure the proper cancellation between PDF factors for branchings in the unordered region. However, (A7) does not have a direct correspondence to any term in the DGLAP equation reformulated as a backwards evolution [39].

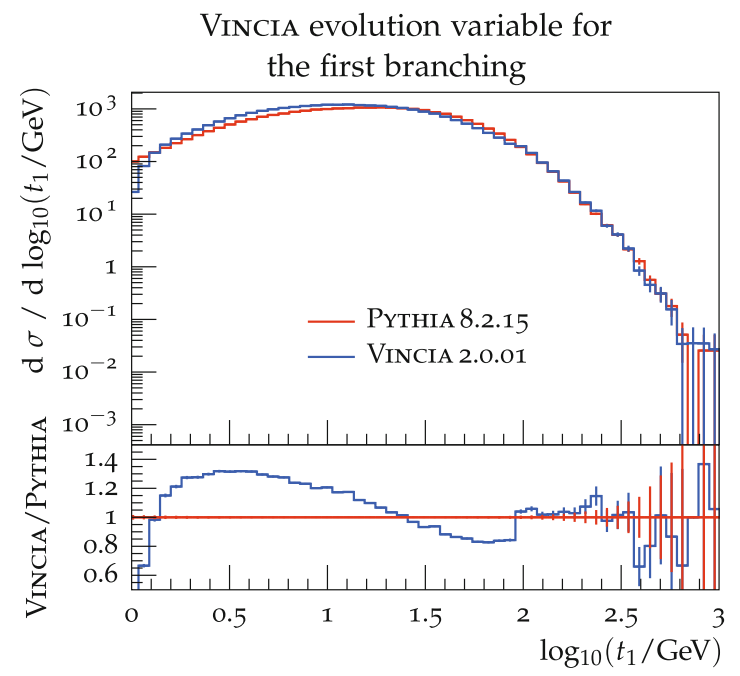

\section{b. Missing evolution and configurations}

For low multiplicities, all partons in the system are treated as unordered and explore their phase space up to the kinematics limit. However, starting for higher multiplicities, "ordered partons" are present which restart their evolution at the Markovian scale. By definition, this scale is smaller or equal to the scale of the last branching. The allowed branching range of "ordered partons" is therefore more restricted than in an ordered shower.

As with every parton shower that only contains QCD splittings, certain flavor configurations cannot be reached, independent of kinematic constraints. One such example is $q \bar{q} \rightarrow W q^{\prime} \bar{q}^{\prime \prime}$, where the $W$ boson can only be radiated off the final-state legs. To include such a configuration within the MECs method an electroweak shower is necessary.

\section{A 2. The treatment of hard jets}

To avoid the concept of "power showers" and simultaneously allow jets with scales $t>t_{\text {fac }}$, VINCIA distinguishes between non-QCD and QCD processes. The latter category covers all hard processes with partons in the final state (except partons arising from resonance decay).

In non-QCD processes the input events are divided in two samples. The first one is associated with no hard jets, while the second sample contains at least one jet with $t>t_{\text {fac }}$. Because both samples are weighted differently, this introduces a non-smooth transition, see the left panel of Fig. 5. When more branchings are taken into account, the effect is washed out and the step barely visible as shown in the right panel of Fig. 5.

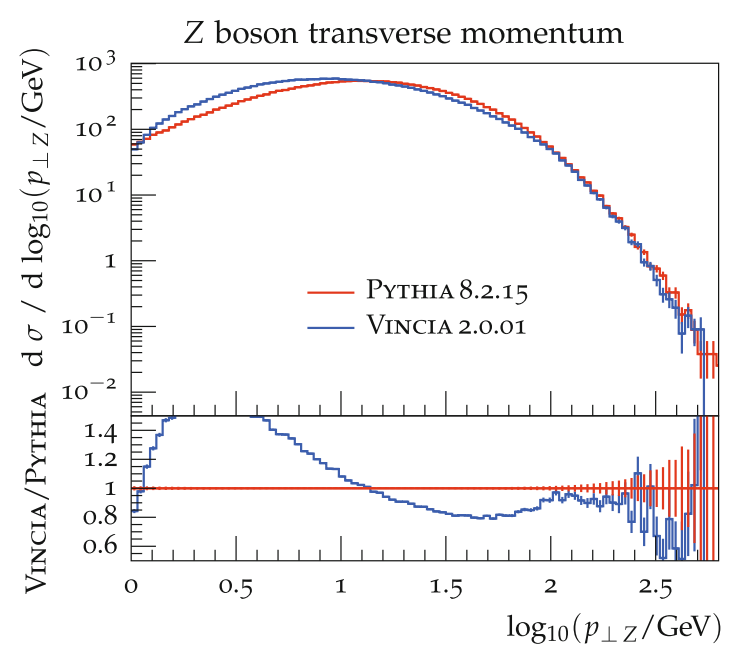

Fig. 5 Distribution of the VINCIA evolution variable after the first branching $(l e f t)$ and the $Z$ boson transverse momentum $(r i g h t)$ for $p p \rightarrow Z+$ jets at parton level 

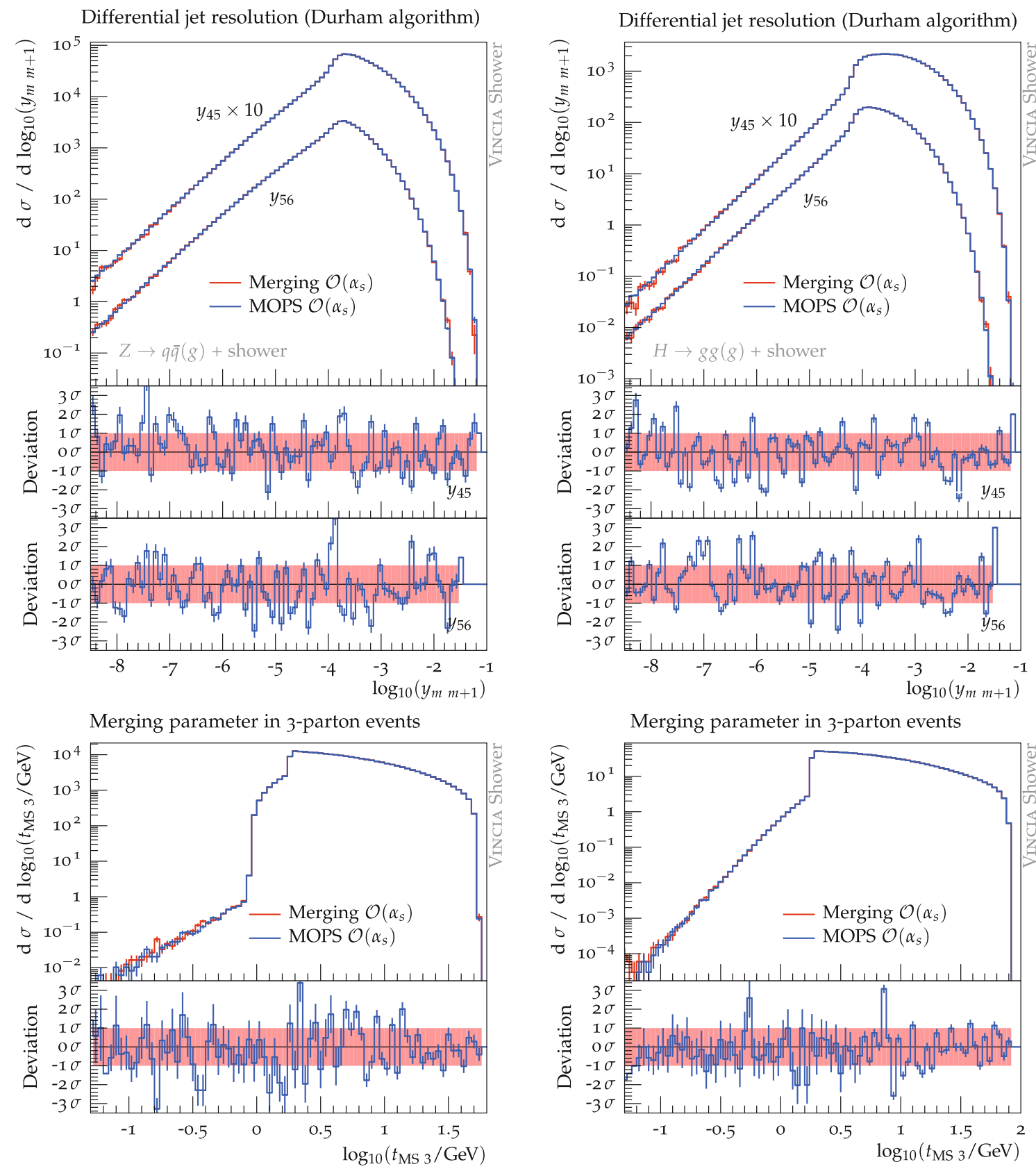

(a) $e^{+} e^{-} \rightarrow Z \rightarrow$ jets

(b) $\tau^{+} \tau^{-} \rightarrow H \rightarrow$ gluons

Fig. 6 Parton-level results: the distribution of the merging scale in exclusive 3-parton events (bottom) and the logarithmic distributions of differential jet resolutions (top). Merged predictions with a merging-scale value of $5 \mathrm{GeV}$ are compared to predictions with the MOPS method

The first emission off a QCD $2 \rightarrow 2$ process is treated similar to the procedure summarized in Appendix A 1: all partons are allowed to explore their full phase space, but with a suppression of

$P_{\text {imp }}\left(t_{\text {fac }}, t\left(\Phi_{1} / \Phi_{0}\right)\right)=\frac{t_{\text {fac }}}{t_{\text {fac }}+t\left(\Phi_{1} / \Phi_{0}\right)}$.

Here the factorization scale replaces the Markovian reference scale. This leads to similar, leftover no-emission probabilities from unordered regions as discussed in Appendix A 1.

\section{Appendix B: Validation of matrix-element corrections for ordered emissions in VINCIA}

In this section we validate the numerical implementation of the MOPS method in VINCIA by comparing it to merged predictions using the CKKW-L merging implementation in Pythia8 [28] applied to VINCIA. For the latter we define the merging scale as the minimum of all evolution scales, $t_{\mathrm{MS}}=\min _{i}\left(t\left(\Phi_{n+1} / \Phi_{n}^{i}\right)\right)$. No color information is used to 
Differential $k_{\perp}$-jet resolution $(R=0.4)$
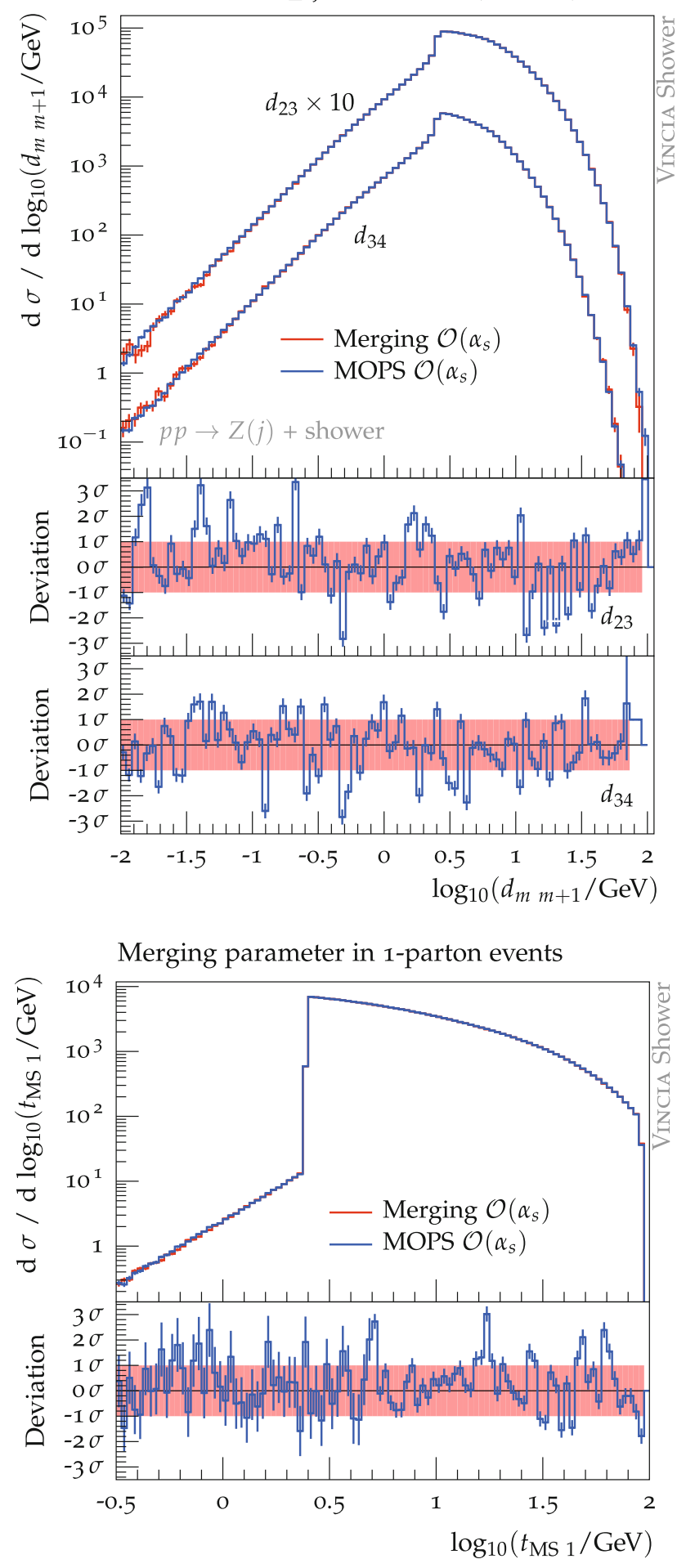

Fig. 7 Parton-level results for $p p \rightarrow Z+$ jets: the distribution of the merging scale in exclusive 1-parton events (bottom) and the logarithmic distributions of differential jet resolutions (top). Merged predictions with a merging-scale value of $5 \mathrm{GeV}$ are compared to predictions with the MOPS method

find possible clusterings. For the validation we use partonlevel results with a fixed $\alpha_{s}$ for both methods and do not include events that cannot be reproduced by VINCIA with an
Differential jet resolution (Durham algorithm)
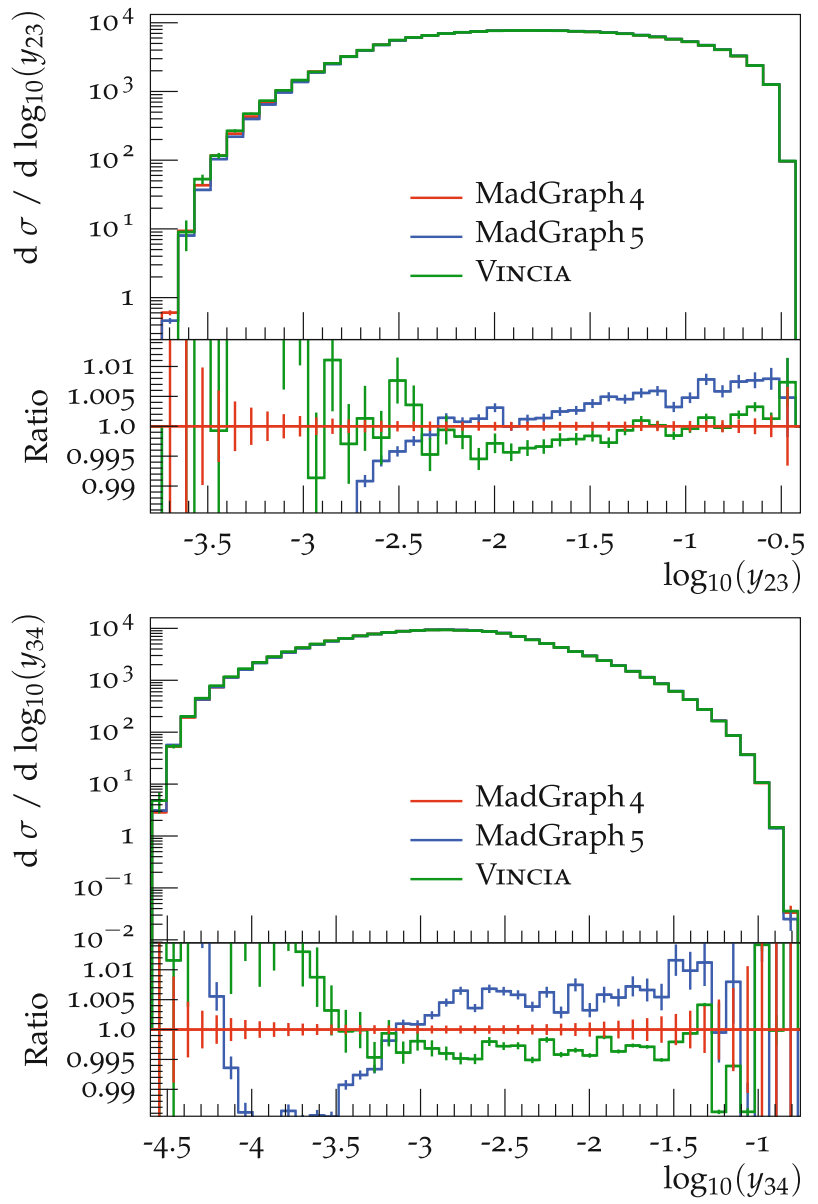

Merging parameter in 4-parton events

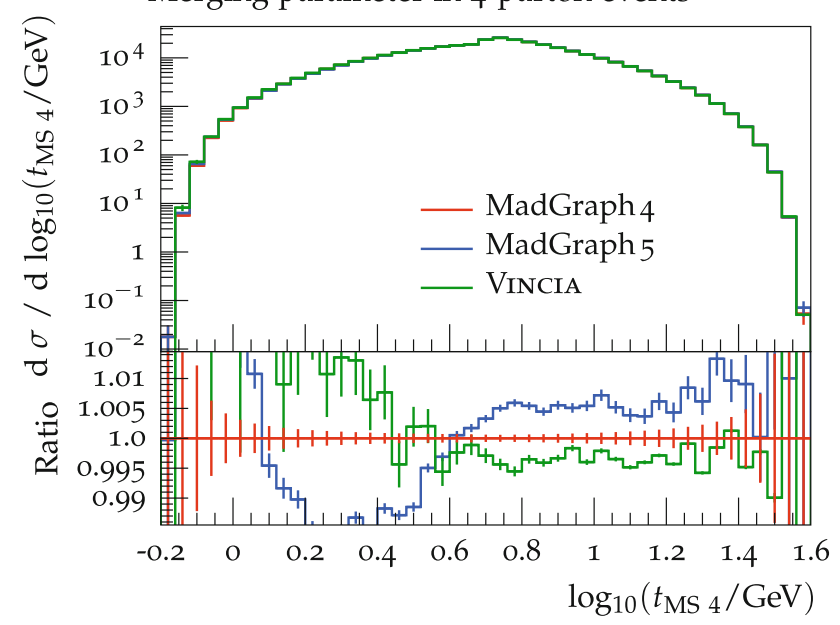

Fig. 8 Parton-level results for $e^{+} e^{-} \rightarrow Z \rightarrow q \bar{q} g g$ : the distribution of the merging scale in exclusive 4-parton events (bottom) and the logarithmic distributions of differential jet resolutions. Comparison of MadGraph 4, MadGraph 5, and VINCIA+MadGraph 4+Rambo

ordered sequence of branching scales. To ensure the same Sudakov factors ME corrections are also applied in the case of merging. 
Figures 6 and 7 show a comparison between the results of the MOPS method and merging including a ME corrected first emission. Each simulation contains at least $10^{8}$ input events generated with MadGraph [61,62]. The lower panels present the deviation between the two methods, normalized to the statistical uncertainty of the merged prediction in the respective bin. As both methods should provide the same result, this distribution should exhibit statistical fluctuations only. Parton-level results for $e^{+} e^{-} \rightarrow Z \rightarrow$ jets and $\tau^{+} \tau^{-} \rightarrow H \rightarrow$ gluons are presented in Fig. 6. The deviation in the lower panels clearly show that both methods are identical up to statistical fluctuations.

Similar plots are shown for on-shell Z-boson production in Fig. 7. Note that we exclude branchings with scales above the factorization scale for comparison purposes. This is necessary due to how such emissions are generated in VINCIA, see Appendix A 2.

When correcting the second emission, we expect slight mismatches between the predictions of the two methods. The matrix elements in VINCIA are taken from MadGraph 4. It would thus be preferable to use MadGraph 4 input for the merging. However, MadGraph 4 is no longer developed and does not allow for linking against LHAPDF 5 [63], while PYTHIA8 requires LHAPDF 5 or higher. Thus, using the same PDF set for hadronic initial states means that the input for merging was generated with MadGraph 5. MadGraph 4 and 5 exhibit shape and normalization differences at the (sub-)percent level in the observables investigated for the validation, as discussed in the following. As an example, we compare the ME output of MadGraph 4 and 5 for $e^{+} e^{-} \rightarrow Z \rightarrow q \bar{q} g g$ with a cut on the invariant mass of jet pairs, $m_{j j} \geq 5 \mathrm{GeV}$. We further include curves for the VINCIA matrix element integrated with RAMBO [64] (an implementation of which is included in VINCIA) and normalized to the MadGraph 4 cross section, as we are mainly interested in shape differences. The results are shown in Fig. 8. The ratio plots shown in the lower panels reveal differences between all three predictions, mostly at the level of around $0.5 \%$. While those mismatches are irrelevant in practical studies, they deteriorate the quality the validation. Nevertheless the results of the validation are satisfactory. When correcting the third emission, we anticipate further differences between the two methods. In VINCIA, the color matrices for matrix elements with two identical quark pairs and at least one gluon are decomposed by hand; see [38]. Therefore, higher orders cannot be validated at the same level as the first order.

In Fig. 9 we show a comparison of merging and the MOPS method for three corrected emissions. The lower panels show the ratio of predictions with the MOPS method to merged results. Small deviations between the two methods are visible at large scales. Considering that the differences are at most $3 \%$, and that we expect some mismatches, and that the differences are mostly in a region where non-shower states have a very large impact (cf. Fig. 3), we find the methods in good agreement.

\section{Appendix C: Identifying and removing the overlap between states with different multiplicities}

As discussed in Sects. 4 and 5, overlap between (the shower off) non-shower states with different parton multiplicities exists and has to be removed. In this section we briefly explain, for interested readers and practitioners, how different states are treated to remove potential overlap.

$+\mathbf{0}$-particle states: The shower is started at the factorization scale $t_{\mathrm{fac}}$ of the Born state and no further restrictions apply.

+1-particle states: Only events where all scales $t_{1}$ exceed the factorization scale, $t_{1}>t_{\mathrm{fac}}$, are taken into account. After a path is chosen, the shower off the +1 -particle state starts at the scale $t_{1}$.

+2-particle states: To avoid overlap with the shower off non-shower +1 -particle states, an ordering of the clustering scales with respect to the factorization scale is not checked. Only events, where $t_{2}>t_{1}$ holds for all paths, are taken into account and the effective scale $t_{2}^{\text {eff }}$ is calculated. If $t_{\text {fac }}>t_{2}^{\text {eff }}$ a Sudakov factor $\Delta\left(t_{\mathrm{fac}}, t_{2}^{\text {eff }}\right)$ is attached by trial-showering the clustered Born state. The shower off the +2 -particle state starts at $t_{2}^{\text {eff }}$.

+ -particle states $(n \geq 3)$ : As for the non-shower +2 particle states, an ordering of the clustering scales with respect to the factorization scale is not checked. Only events without an ordered path are taken into account. The effective scales $t_{2}^{\text {eff }}, t_{3}^{\text {eff }}, \ldots t_{n}^{\text {eff }}$ are calculated and the smallest $k \in\{2 \ldots n\}$ which leads to an ordered sequence of scales, $t_{k}^{\text {eff }}>t_{k+1}>\cdots>t_{n}$, is found. If $k \leq n-2$, the event is removed from consideration due to overlap with showering lower-multiplicity non-shower states, see Sect. 5. If $k=n-1$, i.e. $t_{n-1}^{\text {eff }}>t_{n}$, the event is removed, if the clustered $+(n-1)$-particle state is itself a non-shower state. For events that are not rejected we chose one of the paths for which $t_{n-1}^{\text {eff }}>t_{n}$ holds and attached the Sudakov factors $\Delta\left(t_{\text {fac }}, t_{n-1}^{\text {eff }}\right) \Delta\left(t_{n-1}^{\text {eff }}, t_{n}\right)$. The shower off the $+n$-particle state starts at $t_{n}$. If no scale hierarchy is found, the event is retained, the Sudakov factor $\Delta\left(t_{\mathrm{fac}}, t_{n}^{\text {eff }}\right)$ is attached, and the $+n$-particle states is showered from $t_{n}^{\text {eff }}$. 

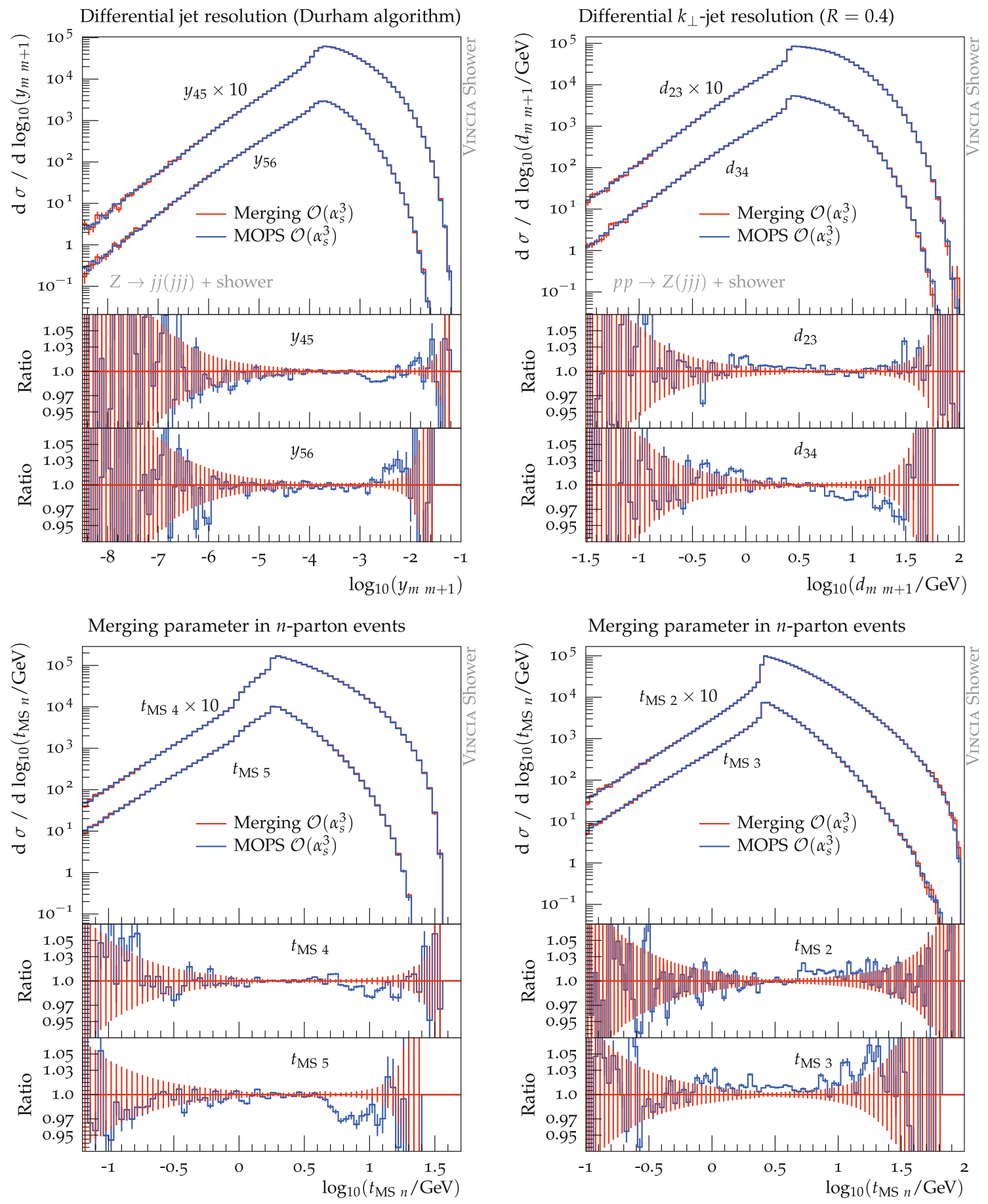

(a) $e^{+} e^{-} \rightarrow Z \rightarrow$ jets

(b) $p p \rightarrow Z+$ jets

Fig. 9 Parton-level results: the distribution of the merging scale in exclusive 4- and 5-parton events (bottom) and the logarithmic distributions of differential jet resolutions (top). Merged predictions with a merging-scale value of $5 \mathrm{GeV}$ are compared to predictions with the MOPS method

\section{References}

1. A. Buckley et al., Phys. Rep. 504, 145 (2011). arXiv:1101.2599 [hep-ph]

2. T. Gleisberg, S. Höche, F. Krauss, M. Schönherr, S. Schumann, F. Siegert, J. Winter, JHEP 02, 007 (2009). arXiv:0811.4622 [hep-ph]
3. T. Sjöstrand, S. Ask, J.R. Christiansen, R. Corke, N. Desai, P. Ilten, S. Mrenna, S. Prestel, C.O. Rasmussen, P.Z. Skands, Comput. Phys. Commun. 191, 159 (2015). arXiv:1410.3012 [hep-ph]

4. M. Bähr et al., Eur. Phys. J. C 58, 639 (2008), arXiv:0803.0883 [hep-ph]

5. S. Frixione, B.R. Webber, JHEP 0206, 029 (2002). arXiv:hep-ph/0204244 
6. P. Nason, JHEP 11, 040 (2004). arXiv:hep-ph/0409146

7. S. Frixione, P. Nason, C. Oleari, JHEP 11, 070 (2007). arXiv:0709.2092 [hep-ph]

8. S. Frixione, F. Stoeckli, P. Torrielli, B.R. Webber, JHEP 1101, 053 (2011). arXiv: 1010.0568 [hep-ph]

9. P. Torrielli, S. Frixione, JHEP 04, 110 (2010). arXiv:1002.4293 [hep-ph]

10. S. Alioli, P. Nason, C. Oleari, E. Re, JHEP 06, 043 (2010). arXiv:1002.2581 [hep-ph]

11. S. Höche, F. Krauss, M. Schönherr, F. Siegert, JHEP 04, 024 (2011). arXiv:1008.5399 [hep-ph]

12. S. Höche, F. Krauss, M. Schönherr, F. Siegert, JHEP 09, 049 (2012). arXiv:1111.1220 [hep-ph]

13. S. Plätzer, S. Gieseke, Eur. Phys. J. C 72, 2187 (2012). arXiv:1109.6256 [hep-ph]

14. J. Alwall, R. Frederix, S. Frixione, V. Hirschi, F. Maltoni, O. Mattelaer, H.S. Shao, T. Stelzer, P. Torrielli, M. Zaro, JHEP 07, 079 (2014). arXiv:1405.0301 [hep-ph]

15. S. Jadach, W. Placzek, S. Sapeta, A. Siodmok, M. Skrzypek, JHEP 10, 052 (2015). arXiv:1503.06849 [hep-ph]

16. M. Czakon, H.B. Hartanto, M. Kraus, M. Worek, JHEP 06, 033 (2015). arXiv:1502.00925 [hep-ph]

17. S. Catani, F. Krauss, R. Kuhn, B.R. Webber, JHEP 11, 063 (2001). arXiv:hep-ph/0109231

18. M.L. Mangano, M. Moretti, R. Pittau, Workshop on Monte Carlo Generator Physics for Run II at the Tevatron Batavia, Illinois, April 18-20, 2001, Nucl. Phys. B 632, 343 (2002). arXiv:hep-ph/0108069

19. S. Mrenna, P. Richardson, JHEP 05, 040 (2004). arXiv:hep-ph/0312274

20. J. Alwall et al., Eur. Phys. J. C 53, 473 (2008). arXiv:0706.2569 [hep-ph]

21. K. Hamilton, P. Richardson, J. Tully, JHEP 11, 038 (2009). arXiv:0905.3072 [hep-ph]

22. K. Hamilton, P. Nason, JHEP 06, 039 (2010). arXiv:1004.1764 [hep-ph]

23. S. Höche, F. Krauss, M. Schonherr, F. Siegert, JHEP 08, 123 (2011). arXiv:1009.1127 [hep-ph]

24. N. Lavesson, L. Lönnblad, JHEP 12, 070 (2008). arXiv:0811.2912 [hep-ph]

25. L. Lönnblad, S. Prestel, JHEP 02, 094 (2013). arXiv:1211.4827 [hep-ph]

26. L. Lönnblad, JHEP 05, 046 (2002). arXiv:hep-ph/0112284

27. N. Lavesson, L. Lönnblad, JHEP 07, 054 (2005). arXiv:hep-ph/0503293

28. L. Lönnblad, S. Prestel, JHEP 03, 019 (2012). arXiv:1109.4829 [hep-ph]

29. S. Plätzer, JHEP 08, 114 (2013). arXiv:1211.5467 [hep-ph]

30. T. Gehrmann, S. Höche, F. Krauss, M. Schonherr, F. Siegert, JHEP 01, 144 (2013). arXiv:1207.5031 [hep-ph]

31. S. Höche, F. Krauss, M. Schonherr, F. Siegert, JHEP 04, 027 (2013). arXiv:1207.5030 [hep-ph]

32. L. Lönnblad, S. Prestel, JHEP 03, 166 (2013). arXiv:1211.7278 [hep-ph]

33. R. Frederix, S. Frixione, JHEP 12, 061 (2012). arXiv:1209.6215 [hep-ph]

34. S. Alioli, C.W. Bauer, C.J. Berggren, A. Hornig, F.J. Tackmann, C.K. Vermilion, J.R. Walsh, S. Zuberi, JHEP 09, 120 (2013). arXiv:1211.7049 [hep-ph]
35. J. Bellm, S. Gieseke, S. Plätzer, (2017). arXiv: 1705.06700 [hep-ph]

36. J.R. Christiansen, S. Prestel, Eur. Phys. J. C 76, 39 (2016). arXiv: 1510.01517 [hep-ph]

37. W.T. Giele, D.A. Kosower, P.Z. Skands, Phys. Rev. D 84, 054003 (2011). arXiv:1102.2126 [hep-ph]

38. N. Fischer, S. Prestel, M. Ritzmann, P. Skands, Eur. Phys. J. C 76, 589 (2016). arXiv: 1605.06142 [hep-ph]

39. T. Sjöstrand, Phys. Lett. B 157, 321 (1985)

40. G. Marchesini, B.R. Webber, Nucl. Phys. B 310, 461 (1988)

41. S. Höche, S. Prestel, Eur. Phys. J. C 75, 461 (2015). arXiv:1506.05057 [hep-ph]

42. D.A. Kosower, Phys. Rev. D 57, 5410 (1998). arXiv:hep-ph/9710213

43. A. Gehrmann-De Ridder, T. Gehrmann, E.W.N. Glover, JHEP 09, 056 (2005). arXiv:hep-ph/0505111

44. M. Bengtsson, T. Sjöstrand, Phys. Lett. B 185, 435 (1987)

45. M. Bengtsson, T. Sjöstrand, Nucl. Phys. B 289, 810 (1987)

46. G. Miu, T. Sjöstrand, Phys. Lett. B 449, 313 (1999). arXiv:hep-ph/9812455

47. M.H. Seymour, Comput. Phys. Commun. 90, 95 (1995). arXiv:hep-ph/9410414

48. G. Parisi, R. Petronzio, Nucl. Phys. B 154, 427 (1979)

49. S. Frixione, P. Nason, G. Ridolfi, Nucl. Phys. B 542, 311 (1999). arXiv:hep-ph/9809367

50. C.F. Berger, Z. Bern, L.J. Dixon, F. Febres Cordero, D. Forde, T. Gleisberg, H. Ita, D.A. Kosower, D. Maitre, Phys. Rev. D 80, 074036 (2009). arXiv:0907.1984 [hep-ph]

51. A. Buckley, J. Butterworth, L. Lönnblad, D. Grellscheid, H. Hoeth, J. Monk, H. Schulz, F. Siegert, Comput. Phys. Commun. 184, 2803 (2013). arXiv:1003.0694 [hep-ph]

52. G. Aad et al. (ATLAS), JHEP 07, 032 (2013). arXiv:1304.7098 [hep-ex]

53. G. Aad et al. (ATLAS), JHEP 09, 145 (2014). arXiv:1406.3660 [hep-ex]

54. S. Chatrchyan et al. (CMS), Phys. Lett. B 722, 238 (2013). arXiv:1301.1646 [hep-ex]

55. V. Khachatryan et al. (CMS), Phys. Rev. D 91, 052008 (2015). arXiv: 1408.3104 [hep-ex]

56. R.D. Ball, V. Bertone, F. Cerutti, L. Del Debbio, S. Forte, A. Guffanti, J.I. Latorre, J. Rojo, M. Ubiali (NNPDF), Nucl. Phys. B 855, 153 (2012). arXiv:1107.2652 [hep-ph]

57. H.T. Li, P. Skands, Phys. Lett. B 771, 59 (2017). arXiv:1611.00013 [hep-ph]

58. S. Höche, S. Prestel, (2017). arXiv:1705.00742 [hep-ph]

59. S. Höche, F. Krauss, S. Prestel, (2017). arXiv:1705.00982 [hep-ph]

60. R.K. Ellis, W.J. Stirling, B.R. Webber, Camb. Monogr. Part. Phys. Nucl. Phys. Cosmol. 8, 176 (1996)

61. J. Alwall, P. Demin, S. de Visscher, R. Frederix, M. Herquet, F. Maltoni, T. Plehn, D.L. Rainwater, T. Stelzer, JHEP 09, 028 (2007). arXiv:0706.2334 [hep-ph]

62. J. Alwall, M. Herquet, F. Maltoni, O. Mattelaer, T. Stelzer, JHEP 06, 128 (2011). arXiv:1106.0522 [hep-ph]

63. M.R. Whalley, D. Bourilkov, R.C. Group, in HERA and the LHC: A Workshop on the Implications of HERA for LHC Physics. Proceedings, Part B, p. 575 (2005). arXiv:hep-ph/0508110

64. R. Kleiss, W.J. Stirling, S.D. Ellis, Comput. Phys. Commun. 40, 359 (1986) 